\title{
Modelos de Regressão Beta-Binomial/Poisson para Contagens Bivariadas
}

\author{
Mayra Ivanoff Lora
}

\author{
DissertaçÃo Apresentada ao \\ Instituto DE MATEMÁtica E Estatística \\ DA Universidade de SÃo Paulo \\ PARA OBTENÇÃo DO TÍtUlo DE \\ Mestre em Estatística
}

\section{Área de Concentração: Estatística \\ Orientador: Prof. Dr. Julio da Motta Singer}

São Paulo, abril de 2004 


\title{
Modelos de Regressão Beta-Binomial/Poisson para Contagens Bivariadas
}

\author{
Este exemplar corresponde à redação \\ final da dissertação devidamente corrigida \\ e defendida por Mayra Ivanoff Lora \\ e aprovada pela comissão julgadora.
}

São Paulo, 01 de abril de 2004.

Banca examinadora:

- Prof. Dr. Julio da Motta Singer (orientador) - IME-USP

- Prof. Dr. Carlos Alberto de Bragança Pereira - IME-USP

- Profa. Dra. Linda Lee Ho - EP-USP 


\section{Agradecimentos}

Ao Professor Julio da Motta Singer, com quem tive o privilégio de trabalhar, pelo apoio, orientação e amizade.

À Professora Linda Lee Ho, pela ajuda com a Poisson e pelo apoio e amizade desde o TF.

Ao Professor Clóvis de Araújo Peres, por todo incentivo e amizade.

Aos amigos do IME, pelas muitas horas de estudo e companheirismo, não podendo deixar de citar o Cléber, amigo desde os tempos de ouvinte.

Aos amigos do "osmanoeasminadapoli", por todos os emails recebidos cada vez que um modelo rodava.

Ao Rodrigo, pelos momentos alegres entre tantas listas.

À minha mãe e minha vó, pelo incentivo, apoio e carinho inesgotáveis. 


\section{Resumo}

Propomos um modelo Beta-Binomial/Poisson para dados provenientes de um estudo com doentes de Parkinson, que consistiu em contar durante um minuto quantas tarefas foram realizadas e destas, quantas de maneira correta, antes e depois de um treinamento. O objetivo era verificar se o treinamento aumentava o número de tentativas e a porcentagem de acerto, o que destaca o aspecto bivariado do problema. Esse modelo considera tal aspecto, usa uma distribuição mais adequada a dados de contagem e ainda suporta a sobredispersão presente nos dados. Obtemos estimadores de máxima verossimilhança dos parâmetros utilizando um algoritmo de Newton-Raphson. Ilustramos a aplicação da metodologia desenvolvida aos dados estudo. 


\section{Abstract}

We propose a Beta-Binomial/Poisson model to the data from a study with Parkinson disease patients, which consisted in counting for one minute how many trials were attempted and how many of them were successful, before and after a training period. The main goal was to check if training increased the number of trials and success probability, which emphasizes the bivariate aspect of the problem. This model takes this aspect into account, uses a distribution which is usually more adequate to count data and supports the overdispersion present in the data. We obtain the maximum likelihood estimators using a Newton-Raphson algorithm. For illustration, the methodology is applied to the data from study. 


\section{Índice}

1 Introdução $\quad 2$

2 Modelo Beta-Binomial/Poisson $\quad 7$

2.1 Estimação sob o modelo Beta-Binomial . . . . . . . . . . . . . . . . . . . 10

2.2 Estimação sob o modelo Poisson Tetravariado . . . . . . . . . . . . . . . . 14

3 Análise dos dados $\quad 19$

3.1 Modelagem do número de acertos . . . . . . . . . . . . . . . . . . . . . 19

3.2 Modelagem do total de tentativas . . . . . . . . . . . . . . . 25

3.3 Resultados finais e sugestões . . . . . . . . . . . . . . . . . . . 32

$\begin{array}{ll}\text { A Correlações amostrais } & 35\end{array}$

B Expressões das derivadas do modelo Beta-Binomial/Poisson 39

B.1 Modelo Beta-Binomial (2.9) f . . . . . . . . . . . . . . . . . . . 39

B.1.1 Primeiras derivadas . . . . . . . . . . . . . . . . . . . 39

B.1.2 Segundas derivadas . . . . . . . . . . . . . . . . . . . . 39

B.2 Modelo Poisson Tetravariado completo (3.1) . . . . . . . . . . . . . . . . 40

B.2.1 Primeiras derivadas . . . . . . . . . . . . . . . . . . 40

B.2.2 Segundas derivadas . . . . . . . . . . . . . . . . . 40

B.3 Modelo Poisson Tetravariado final (3.2) . . . . . . . . . . . . . . . . 42

B.3.1 Primeiras derivadas . . . . . . . . . . . . . . . 42

B.3.2 Segundas derivadas . . . . . . . . . . . . . . . . . . . 43

C Códigos dos processos iterativos $\quad 46$

C.1 Modelo Beta-Binomial . . . . . . . . . . . . . . . . . 46 
C.2 Modelos Poisson Tetravariado completo (3.1) e final (3.2) . . . . . . . . 46

$\begin{array}{lll}\text { D Resultados das simulações } & 48\end{array}$

Referências Bibliográficas $\quad 52$ 


\section{Capítulo 1}

\section{Introdução}

A doença de Parkinson é uma das patologias degenerativas mais freqüentes do sistema nervoso central, caracterizando-se principalmente por sintomas motores, sendo o tremor de repouso, na maioria dos casos, o sintoma inicial e a bradicinesia (dificuldade global para iniciar movimentos), o sintoma que limita as atividades da vida diária do paciente. O início da doença ocorre em geral entre 50 e 70 anos, sem diferenças entre sexos.

Neste contexto, o objetivo de um estudo realizado no Laboratório de Aprendizado Processual do Departamento de Fisioterapia, Fonoaudiologia e Terapia Ocupacional da Faculdade de Medicina da Universidade de São Paulo é verificar se o treinamento de atividades motoras pode interferir na bradicinesia, possibilitando uma melhora nas condições motoras dos doentes. Foram selecionados 25 indivíduos com diagnóstico confirmado de doença de Parkinson (grupo experimental) e um grupo controle de 21 pessoas, sem antecedentes de alterações neurológicas detectáveis. Os pacientes do grupo experimental foram classificados de acordo com o estágio da doença em duas categorias (estágio inicial e estágio avançado). Para mais detalhes, ver Singer et al. (2002).

O estudo envolveu a repetição de duas seqüências predeterminadas (seqüências 1 e 2) de oposição dos dedos, ou seja, conjunto de toques do polegar em um dos outros quatro dedos, numerados de 1 a 4, do indicador ao dedo mínimo. Durante a execução das seqüências, foram registrados todos os movimentos por intermédio de uma luva e de um cabo ligado a um computador; informações sobre o número de seqüências realizadas de 
maneira correta e errada foram armazenadas.

Numa avaliação inicial, os indivíduos realizaram cada uma das seqüências durante um minuto, com as duas mãos. Após essa avaliação inicial, todos os indivíduos participaram de um treinamento de quatro semanas, praticando apenas uma das seqüências com uma das mãos, de maneira que metade dos participantes praticou a seqüência 1 e a outra metade, a seqüência 2 .

Tanto no grupo controle como no experimental, metade dos participantes realizou o treinamento com a mão preferencial e a outra metade, com a não preferencial, formando inicialmente quatro subgrupos. Para o grupo controle, mão preferencial é a esquerda para canhotos e a direita para destros; já para o grupo experimental, mão preferencial é a menos afetada pela doença. Metade dos indivíduos de cada um dos quatro subgrupos treinou a seqüência 1 e a outra metade treinou a 2, formando assim oito subgrupos.

Após o treinamento, os indivíduos foram reavaliados da mesma maneira que no instante inicial - realizando as duas seqüências por um minuto com as duas mãos. Foram registrados, da mesma maneira, o número de seqüências corretas e erradas. Ainda durante a avaliação final, os participantes repetiram as seqüências (os dois tipos com as duas mãos), porém sob condições que dificultassem a repetição. Essa condições foram:

- sem a indicação visual do número do dedo na luva (condição 1);

- sem olhar para a mão (condição 2);

- com a realização de uma tarefa distratora concorrente - listar nomes próprios que começassem com determinada letra, sem repetições (condição 3);

- com a realização de tarefa motora concorrente - conduzir bolinhas em uma trajetória fixa (condição 4).

Devido a dificuldades em contar o número de seqüências corretas e erradas com o programa usado para registrar todos os movimentos, não foram disponibilizadas as contagens para a mão não treinada dos participantes. Desta maneira, para cada participante obtiveram-se as contagens para a mão treinada, com as seqüências 1 e 2 , antes e depois do treinamento, e sob as quatro situações dificultadoras. 
Na Tabela 1.1, estão apresentadas as médias e desvios-padrão das contagens de acertos e totais das avaliações inicial e final, para as seqüências treinada e controle, para os 6 grupos de estudo, formados pelas combinações de estágio da doença (sem doença, estágio inicial e avançado) e uso da mão preferencial (sim ou não). Além disso, no Apêndice A estão apresentadas as correlações entre as contagens de acertos e totais, para as seqüências treinada e controle, antes e depois do treinamento. Os resultados sugerem sobredispersão nas contagens.

Os objetivos desse estudo são verificar se o treinamento melhora o desempenho dos participantes na realização das seqüências e se essa possível melhora é similar para pacientes doentes e controles. Para tanto, pretende-se avaliar se o treinamento tem efeito no número de tentativas por minuto (agilidade) e na porcentagem de tentativas certas, o que destaca a característica bivariada do estudo. Além disso, há interesse em comparar a mão preferencial com a não preferencial e avaliar se as 4 condições distratoras de fato dificultam o desempenho.

Uma maneira natural para avaliar os dados, considerando simultaneamente o número de acertos e totais, seria criar algum índice que os relacionasse, como a razão entre número de acertos e erros; tal relação não consideraria o total de tentativas, atribuindo, por exemplo, valor 1 para quem acertou uma seqüência e errou outra, e valor 0,98 para quem acertou 98 seqüências e errou 100 e consequentemente indicando uma situação pior para o segundo indivíduo, que tentou um número muito maior de seqüências. Uma outra alternativa seria a razão entre número de acertos (ou erros) e total de seqüências; nesse caso, por exemplo, o mesmo valor seria atribuído para quem tentou 2 vezes e acertou 1, e para quem acertou 50 em 100 seqüências, recaindo em uma situação semelhante à anterior.

A taxa de acertos por unidade de tempo também poderia ser analisada, já que todos os participantes executaram as seqüências durante um minuto. Tal análise também não permitiria verificar, por exemplo, se um determinado grupo é mais ágil que outro (ou seja, se executa mais repetições), por não considerar o total de tentativas realizadas.

Uma outra maneira de analisar os dados seria avaliar os resultados de contagens corretas e totais separadamente. Para tanto, poderiam ser criadas variáveis que repre- 
Tabela 1.1: Médias e desvios padrão para o número de acertos e total de tentativas dos grupos de estudo nas diferentes situações de realização das seqüências

\begin{tabular}{cccccc}
\hline Estágio & Avaliação & Mão & Seqüência & Acertos (DP) & Totais (DP) \\
\hline controle & inicial & preferencial & treinada & $17,1(8,5)$ & $17,9(8,9)$ \\
controle & inicial & preferencial & controle & $17,1(7,0)$ & $18,6(6,8)$ \\
controle & inicial & não & treinada & $17,1(6,1)$ & $19,5(7,3)$ \\
controle & inicial & não & controle & $18,1(5,2)$ & $20,9(6,9)$ \\
controle & final & preferencial & treinada & $32,7(11,8)$ & $33,1(11,5)$ \\
controle & final & preferencial & controle & $20,9(9,5)$ & $26,1(6,7)$ \\
controle & final & não & treinada & $32,8(8,6)$ & $34,4(8,5)$ \\
controle & final & não & controle & $24,2(5,0)$ & $28,6(6,2)$ \\
\hline inicial & inicial & preferencial & treinada & $12,0(4,8)$ & $13,5(4,8)$ \\
inicial & inicial & preferencial & controle & $13,7(4,9)$ & $16,3(6,7)$ \\
inicial & inicial & não & treinada & $10,7(4,5)$ & $13,6(3,3)$ \\
inicial & inicial & não & controle & $12,0(4,2)$ & $14,6(3,0)$ \\
inicial & final & preferencial & treinada & $20,2(3,1)$ & $21,8(1,7)$ \\
inicial & final & preferencial & controle & $13,2(5,5)$ & $16,8(6,6)$ \\
inicial & final & não & treinada & $20,1(5,8)$ & $20,4(6,3)$ \\
inicial & final & não & controle & $15,3(10,6)$ & $20,3(10,8)$ \\
\hline avançado & inicial & preferencial & treinada & $4,6(3,4)$ & $7,9(3,8)$ \\
avançado & inicial & preferencial & controle & $4,8(4,7)$ & $7,1(3,4)$ \\
avançado & inicial & não & treinada & $13,5(9,6)$ & $15,5(7,6)$ \\
avançado & inicial & não & controle & $8,3(8,5)$ & $12,5(3,9)$ \\
avançado & final & preferencial & treinada & $13,5(9,5)$ & $14,9(8,8)$ \\
avançado & final & preferencial & controle & $7,4(8,7)$ & $11,9(8,2)$ \\
avançado & final & não & treinada & $22,5(8,7)$ & $23,8(8,7)$ \\
avançado & final & não & controle & $5,8(5,6)$ & $12,8(3,5)$ \\
\hline & & & & &
\end{tabular}


sentassem a diferença entre duas avaliações que se deseja comparar (como a diferença entre os totais de tentativas final e inicial), e então avaliar os efeitos de estágio e uso da mão preferencial nas contagens das seqüências treinada e controle. Ao analisar o estudo dessa maneira não se leva em conta seu aspecto multivariado - contagens de acertos e totais, possivelmente correlacionadas. Alternativamente, poder-se-ia considerar um modelo multivariado para a resposta dos indivíduos, não separando acertos e totais, nem efetuando as diferenças entre avaliações final e inicial; dessa maneira o aspecto multivariado do problema se conservaria. Tal análise seria relativamente simples, apresentando solução implementada em software estatístico (como o SAS), e respondendo aos objetivos do estudo de maneira mais adequada do que a primeira proposta (por ser uma análise simultânea de acertos e erros, permitir comparar a agilidade dos participantes e estimar as covariâncias de interesse, por exemplo). Por outro lado, a distribuição Normal, usualmente empregada nesses casos, não é adequada para dados de contagem, consistindo apenas em uma aproximação; além disso, outra desvantagem ocorreria ao comparar a porcentagem de acertos (razão entre a estimativa de acertos e de totais) quando seria necessário usar testes aproximados, ou modelos log-lineares, que não estão implementados em software comercial.

Para considerar simultaneamente o aspecto multivariado do estudo e utilizar uma distribuição de probabilidades mais adequada para os dados, poder-se-ia usar uma distribuição de Poisson Multivariada ou Poisson Log-Normal Multivariada (como em Ho e Singer, 1997). Tais distribuições, porém, não admitem incluir o fato de o número de acertos ser sempre menor ou igual ao número total de repetições. Com essa finalidade, consideramos um modelo Binomial/Poisson, condicionando o número de acertos ao número de tentativas. Como esse modelo não contempla a sobredispersão presente nos dados de contagem dos acertos, nem a das contagens totais, incorporamos efeitos aleatórios.

No capítulo 2 é apresentado o modelo estatístico proposto e no capítulo 3 sua aplicação para os dados do estudo. 


\section{Capítulo 2}

\section{Modelo Beta-Binomial/Poisson}

Consideremos o vetor resposta

$$
\mathbf{Y}_{i j k}=\left(X_{i j k I S}, N_{i j k I S}, X_{i j k I C}, N_{i j k I C}, X_{i j k F S}, N_{i j k F S}, X_{i j k F C}, N_{i j k F C}\right)^{\prime}
$$

em que $X_{i j k m n}$ corresponde ao número de seqüências corretas em $N_{i j k m n}$ tentativas, para o $i$-ésimo indivíduo $\left(1,2, \ldots, m_{j}\right)$, com $j$-ésimo nível de gravidade da doença $(0=$ controle, $1=$ estágio inicial, $2=$ estágio avançado), usando a mão $k(1=$ preferencial, $2=$ não preferencial), realizando a seqüência $n$ ( $\mathrm{S}=$ treinada, $\mathrm{C}=$ controle), na avaliação $m$ ( $\mathrm{I}=$ inicial, $\mathrm{F}=$ final). Para a comparação entre a avaliação final e uma das avaliações com condição dificultadora, os valores das contagens podem ser apresentados numa forma vetorial semelhante, alterando os valores para o índice $m$ para $m=\mathrm{F}$ (final), D (condição dificultadora $\mathrm{D}=1,2,3,4)$.

Consideremos também que:

(i) $X_{i j k m n} \mid N_{i j k m n}, \pi_{i j k m n} \sim \operatorname{Binomial}\left(N_{i j k m n}, \pi_{i j k m n}\right)$,

$j=0,1,2, k=1,2, m=I, F, n=S, C$, independentes

(ii) $\pi_{i j k m n} \sim \operatorname{Beta}\left(\mu_{j k m n} / \theta_{j k m n},\left(1-\mu_{j k m n}\right) / \theta_{j k m n}\right)$,

$j=0,1,2, \quad k=1,2, m=I, F, n=S, C$, independentes

(iii) $\left(N_{i j k I S}, N_{i j k I C}, N_{i j k F S}, N_{i j k F C}\right)^{\prime} \sim$

Poisson Tetravariada $\left(\lambda_{j k I S}-\lambda_{j k}, \lambda_{j k I C}-\lambda_{j k}, \lambda_{j k F S}-\lambda_{j k}, \lambda_{j k F C}-\lambda_{j k}, \lambda_{j k}\right)$,

$j=0,1,2, \quad k=1,2$, independentes 
O modelo (2.3) é baseado no caso bivariado apresentado em Holgate (1964); os detalhes da distribuição encontram-se na seção 2.2. Segundo os modelos (2.1) e (2.2), indivíduos do mesmo grupo não têm igual probabilidade de acertar uma seqüência, mas essas probabilidades são geradas a partir de uma mesma distribuição. A parametrização adotada para a distribuição Beta foi sugerida por Gange et al. (1996) para facilitar a estimação via máxima verossimilhança. A relação entre os parâmetros $\mu$ e $\theta$ e os parâmetros usualmente utilizados para a distribuição Beta $(\alpha, \delta)$ (como em Johnson e Kotz, 1969) é dada por

$$
\mu=\frac{\alpha}{\alpha+\delta}, \quad(0<\mu<1) \quad \text { e } \quad \theta=\frac{1}{\alpha+\delta}, \quad(\theta>0) .
$$

Os momentos centrais de primeira e segunda ordem de (2.2) são

$$
\begin{aligned}
& E\left(\pi_{i j k m n}\right)=\mu_{j k m n} \\
& \operatorname{Var}\left(\pi_{i j k m n}\right)=\mu_{j k m n}\left(1-\mu_{j k m n}\right) \theta_{j k m n}\left(1+\theta_{j k m n}\right)^{-1} .
\end{aligned}
$$

De (2.1) e (2.2), pode-se concluir que:

$$
\begin{aligned}
& X_{i j k m n} \mid N_{i j k m n} \sim \text { Beta-Binomial }\left(N_{i j k m n}, \mu_{j k m n}, \theta_{j k m n}\right), \\
& j=0,1,2, k=1,2, \quad m=I, F, n=S, C
\end{aligned}
$$

com

$$
\begin{aligned}
& E\left(X_{i j k m n} \mid N_{i j k m n}\right)=N_{i j k m n} \mu_{j k m n} \\
& \operatorname{Var}\left(X_{i j k m n} \mid N_{i j k m n}\right)=N_{i j k m n} \mu_{j k m n}\left(1-\mu_{j k m n}\right) \frac{\left(1+N_{i j k m n} \theta_{j k m n}\right)}{\left(1+\theta_{j k m n}\right)} .
\end{aligned}
$$

Utilizando o fato que dadas duas variáveis aleatórias $\mathrm{X}$ e $\mathrm{Y}$,

$$
E(X)=E[E(X \mid Y)] \quad \text { e } \quad \operatorname{Var}(X)=\operatorname{Var}[E(X \mid Y)]+E[\operatorname{Var}(X \mid Y)]
$$

os momentos centrais de primeira e segunda ordem para as contagens de acertos são

$$
\begin{gathered}
E\left(X_{i j k m n}\right)=\lambda_{j k m n} \mu_{j k m n} \\
\operatorname{Var}\left(X_{i j k m n}\right)=\lambda_{j k m n} \mu_{j k m n}+\lambda_{j k m n}^{2} \mu_{j k m n}\left(1-\mu_{j k m n}\right) \theta_{j k m n}\left(1+\theta_{j k m n}\right)^{-1}
\end{gathered}
$$




$$
\begin{gathered}
\operatorname{Cov}\left(X_{i j k m n}, N_{i j k m n}\right)=\lambda_{j k m n} \mu_{j k m n} \\
\operatorname{Cov}\left(X_{i j k m n}, X_{i j k m^{\prime} n^{\prime}}\right)=\lambda_{j k} \mu_{j k m n} \mu_{j k m^{\prime} n^{\prime}}, m \neq m^{\prime} \text { e/ou } n \neq n^{\prime},
\end{gathered}
$$

$j=0,1,2, k=1,2, m, m^{\prime}=I, F, n, n^{\prime}=S, C$.

Pode-se notar que o valor esperado do número de acertos é função de dois componentes: o primeiro $\left(\lambda_{j k m n}\right)$ ligado à agilidade e o segundo $\left(\mu_{j k m n}\right)$, à capacidade de acerto.

Analogamente, os momentos centrais de primeira e segunda ordem para as contagens dos totais de tentativas são

$$
\begin{array}{r}
E\left(\begin{array}{c}
N_{i j k I S} \\
N_{i j k I C} \\
N_{i j k F S} \\
N_{i j k F C}
\end{array}\right)=\left(\begin{array}{c}
\lambda_{j k I S} \\
\lambda_{j k I C} \\
\lambda_{j k F S} \\
\lambda_{j k F C}
\end{array}\right) \\
\operatorname{Var}\left(\begin{array}{c}
N_{i j k I S} \\
N_{i j k I C} \\
N_{i j k F S} \\
N_{i j k F C}
\end{array}\right)=\left(\begin{array}{cccc}
\lambda_{j k I S} & \lambda_{j k} & \lambda_{j k} & \lambda_{j k} \\
\lambda_{j k} & \lambda_{j k I C} & \lambda_{j k} & \lambda_{j k} \\
\lambda_{j k} & \lambda_{j k} & \lambda_{j k F S} & \lambda_{j k} \\
\lambda_{j k} & \lambda_{j k} & \lambda_{j k} & \lambda_{j k F C}
\end{array}\right)
\end{array}
$$

Com esse modelo Beta-Binomial/Poisson é possível comparar diretamente o valor esperado da probabilidade de acerto dos diferentes grupos de estudo $\left(\mu_{j k m n}=E\left(\pi_{j k m n}\right)\right)$, além de comparar os valores esperados das agilidades $\left(\lambda_{j k m n}\right)$.

Com base em (2.1), (2.2) e (2.3), a distribuição conjunta das contagens de acertos e totais das duas seqüências nas duas avaliações pode ser escrita como:

$$
\begin{aligned}
& P\left(X_{i j k I S}, N_{i j k I S}, X_{i j k I C}, N_{i j k I C}, X_{i j k F S}, N_{i j k F S}, X_{i j k F C}, N_{i j k F C}\right)= \\
= & \prod_{m=I}^{F} \prod_{n=S}^{C}\left(\int_{0}^{1} P\left(X_{i j k m n} \mid N_{i j k m n}, \pi_{i j k m n}\right) f\left(\pi_{i j k m n}\right) d \pi_{i j k m n}\right) \\
& P\left(N_{i j k I S}, N_{i j k I C}, N_{i j k F S}, N_{i j k F C}\right)= \\
= & \prod_{m=I}^{F} \prod_{n=S}^{C} P\left(X_{i j k m n} \mid N_{i j k m n}\right) P\left(N_{i j k I S}, N_{i j k I C}, N_{i j k F S}, N_{i j k F C}\right) .
\end{aligned}
$$

com $f$ denotando a densidade de (2.2). Como o logaritmo da verossimilhança é dado por

$$
\sum_{j=0}^{2} \sum_{i=1}^{m_{j}} \log P\left(X_{i j k I S}, N_{i j k I S}, X_{i j k I C}, N_{i j k I C}, X_{i j k F S}, N_{i j k F S}, X_{i j k F C}, N_{i j k F C}\right)=
$$




$$
=\sum_{j=0}^{2} \sum_{i=1}^{m_{j}} \sum_{m=I}^{F} \sum_{n=S}^{C} \log P\left(X_{i j k m n} \mid N_{i j k m n}\right)+\sum_{j=0}^{2} \sum_{i=1}^{m_{j}} \log P\left(N_{i j k I S}, N_{i j k I C}, N_{i j k F S}, N_{i j k F C}\right),
$$

pode-se notar que a função log-verossimilhança é composta por dois termos, o primeiro associado à distribuição Beta-Binomial $\left(X_{i j k m n} \mid \mathrm{N}_{i j k m n}\right)$, e o segundo, à Poisson Tetravariada. A estimação dos parâmetros da distribuição Beta-Binomial (2.6) via máxima verossimilhança pode ser feita separadamente da estimação dos parâmetros da distribuição Poisson Tetravariada (2.3). A estimação dos parâmetros do modelo Beta-Binomial será apresentada na seção 2.1 e a estimação dos parâmetros do modelo Poisson Tetravariado, na seção 2.2 .

\subsection{Estimação sob o modelo Beta-Binomial}

Com a parametrização proposta e com (2.5) e (2.8), pode-se notar que o parâmetro $\theta_{j k m n}$, além de estar relacionado à variabilidade da probabilidade de acerto, também está relacionado à sobredispersão para a contagem de acertos. Dessa maneira, quando $\theta=0$, não há sobredispersão e temos $X_{i j k m n} \mid N_{i j k m n} \sim \operatorname{Binomial}\left(N_{i j k m n}, \pi_{j k m n}\right)$, ou seja, o modelo Binomial é um caso particular do Beta-Binomial proposto. Quanto maior o valor de $\theta_{j k m n}$ maior a sobredispersão dos dados, sendo que o valor máximo é dado para $\lim _{\theta \rightarrow \infty} \operatorname{Var}\left(X_{i j k m n} \mid N_{i j k m n}\right)=N_{i j k m n}^{2} \mu_{j k m n}\left(1-\mu_{j k m n}\right)$.

A distribuição Beta-Binomial pode ser escrita como

$$
\begin{gathered}
P\left(X_{i j k m n} \mid N_{i j k m n}\right)= \\
=\Gamma\left(\frac{\mu_{j k m n}}{\theta_{j k m n}}+\frac{1-\mu_{j k m n}}{\theta_{j k m n}}\right)\left[\Gamma\left(\frac{\mu_{j k m n}}{\theta_{j k m n}}\right)\right]^{-1}\left[\Gamma\left(\frac{1-\mu_{j k m n}}{\theta_{j k m n}}\right)\right]^{-1} \Gamma\left(\frac{\mu_{j k m n}}{\theta_{j k m n}}+X_{i j k m n}\right) \\
\Gamma\left(\frac{1-\mu_{j k m n}}{\theta_{j k m n}}+N_{i j k m n}-X_{i j k m n}\right)\left[\Gamma\left(\frac{\mu_{j k m n}}{\theta_{j k m n}}+\frac{1-\mu_{j k m n}}{\theta_{j k m n}}+N_{i j k m n}\right)\right]^{-1}\left(\begin{array}{l}
N_{i j k m n} \\
X_{i j k m n}
\end{array}\right) \\
=\left\{\Gamma\left(\frac{1}{\theta_{j k m n}}\right)\left[\Gamma\left(\frac{1}{\theta_{j k m n}}+N_{i j k m n}\right)\right]^{-1}\right\}\left\{\Gamma\left(\frac{\mu_{j k m n}}{\theta_{j k m n}}+X_{i j k m n}\right)\left[\Gamma\left(\frac{\mu_{j k m n}}{\theta_{j k m n}}\right)\right]^{-1}\right\} \\
\left\{\Gamma\left(\frac{1-\mu_{j k m n}}{\theta_{j k m n}}+N_{i j k m n}-X_{i j k m n}\right)\left[\Gamma\left(\frac{1-\mu_{j k m n}}{\theta_{j k m n}}\right)\right]^{-1}\right\}\left(\begin{array}{l}
N_{i j k m n} \\
X_{i j k m n}
\end{array}\right)
\end{gathered}
$$




$$
\begin{gathered}
=\prod_{u=0}^{N_{i j k m n}-1}\left(1+u \theta_{j k m n}\right)^{-1} \prod_{v=0}^{X_{i j k m n}-1}\left(\mu_{j k m n}+v \theta_{i j k m n}\right) \\
\quad \prod_{w=0}^{N_{i j k m n}-X_{i j k m n}-1}\left(1-\mu_{j k m n}+w \theta_{j k m n}\right)\left(\begin{array}{c}
N_{i j k m n} \\
X_{i j k m n}
\end{array}\right)
\end{gathered}
$$

Na expressão acima, as simplificações para as razões entre duas funções gama (apresentadas entre chaves), fazem sentido quando $N_{i j k m n} \neq 0$ (para a primeira razão), $X_{i j k m n} \neq 0$ (para a segunda razão) e $X_{i j k m n} \neq N_{i j k m n}$ (para a terceira razão). Caso estas condições não estejam satisfeitas, a razão entre as funções gama será igual a um, não alterando o valor da probabilidade de $X_{i j k m n} \mid N_{i j k m n}$.

A função log-verossimilhança (a menos de constantes) é

$$
\begin{aligned}
L & =\sum_{j=0}^{2} \sum_{i=0}^{m_{j}} \sum_{m=I}^{F} \sum_{n=C}^{S} \log P\left(X_{i j k m n} \mid N_{i j k m n}\right)= \\
& =\sum_{j=0}^{2} \sum_{i=0}^{m_{j}} \sum_{m=I}^{F} \sum_{n=C}^{S}\left[\sum_{v=0}^{X_{i j k m n}-1} \log \left(\mu_{j k m n}+v \theta_{j k m n}\right)+\right. \\
& \left.\sum_{w=0}^{N_{i j k m n}-X_{i j k m n}-1} \log \left(1-\mu_{j k m n}+w \theta_{j k m n}\right)-\sum_{u=0}^{N_{i j k m n}-1} \log \left(1+u \theta_{j k m n}\right)\right]
\end{aligned}
$$

Com o objetivo de avaliar os efeitos dos fatores de interesse (estágio da doença e uso da mão preferencial), modelamos os parâmetros $\mu_{j k m n}$ e $\theta_{j k m n}$, por intermédio de

$$
\begin{gathered}
\mu_{j k m n}=\frac{\exp \left(\mathbf{z}_{1 i j k m n}^{\prime} \boldsymbol{\beta}_{1}\right)}{1+\exp \left(\mathbf{z}_{1 i j k m n}^{\prime} \boldsymbol{\beta}_{1}\right)} \\
\theta_{j k m n}=\exp \left(\mathbf{z}_{2 i j k m n}^{\prime} \boldsymbol{\beta}_{2}\right),
\end{gathered}
$$

ou equivalentemente por

$$
\begin{aligned}
& \eta_{1}=\log \left(\frac{\mu_{j k m n}}{1-\mu_{j k m n}}\right)=\mathbf{z}_{1 i j k m n}^{\prime} \boldsymbol{\beta}_{1} \\
& \eta_{2}=\log \left(\theta_{j k m n}\right)=\mathbf{z}_{2 i j k m n}^{\prime} \boldsymbol{\beta}_{2}
\end{aligned}
$$

em que $\mathbf{z}_{1 i j k m n}$ e $\mathbf{z}_{2 i j k m n}$ são vetores de variáveis explicativas associados a cada indivíduo e $\boldsymbol{\beta}_{1}$ e $\boldsymbol{\beta}_{2}$ são vetores de parâmetros. 
Para estimar $\boldsymbol{\beta}_{1}$ e $\boldsymbol{\beta}_{2}$ via máxima verossimilhança consideramos as primeiras derivadas de (2.9), que em notação matricial podem ser expressas como

$$
\frac{\partial L}{\partial \boldsymbol{\beta}_{1}}=\boldsymbol{Z}_{1}^{\prime} \mathbf{W A} \quad \text { e } \quad \frac{\partial L}{\partial \boldsymbol{\beta}_{2}}=\boldsymbol{Z}_{2}^{\prime} \mathbf{T B}
$$

com

$$
\begin{aligned}
& \mathbf{A}=\left(a_{10 k I S}, \ldots, a_{i j k m n}, \ldots, a_{12,2 k F C}\right)^{\prime}, \\
& a_{i j k m n}=\sum_{v=0}^{X_{i j k m n}-1} \frac{1}{\mu_{j k m n}+v \theta_{j k m n}}-\sum_{w=0}^{N_{i j k m n}-X_{i j k m n}-1} \frac{1}{1-\mu_{j k m n}+w \theta_{j k m n}} \\
& \mathbf{B}=\left(b_{10 k I S}, \ldots, b_{i j k m n}, \ldots, b_{12,2 k F C}\right)^{\prime}, \\
& b_{i j k m n}=\sum_{v=0}^{X_{i j k m n}-1} \frac{v}{\mu_{j k m n}+v \theta_{j k m n}}+\sum_{w=0}^{N_{i j k m n}-X_{i j k m n}-1} \frac{w}{1-\mu_{j k m n}+w \theta_{j k m n}} \\
& \mathbf{W}=\operatorname{diag}\left(\mu_{j k m n}\left(1-\mu_{j k m n}\right)\right) \\
& \mathbf{T}=\operatorname{diag}\left(\theta_{j k m n}\right) \\
& \mathbf{Z}_{1}=\left(\mathbf{z}_{110 k I S}^{\prime}, \ldots, \mathbf{z}_{1 i j k m n}^{\prime}, \ldots, \mathbf{z}_{1,12,2 k F C}^{\prime}\right)^{\prime} \\
& \mathbf{Z}_{2}=\left(\mathbf{z}_{210 k I S}^{\prime}, \ldots, \mathbf{z}_{2 i j k m n}^{\prime}, \ldots, \mathbf{z}_{2,12,2 k F C}^{\prime}\right)^{\prime} .
\end{aligned}
$$

As segundas derivadas, podem ser expressas como

$$
\frac{\partial^{2} L}{\partial \boldsymbol{\beta}_{1} \boldsymbol{\beta}_{1}^{\prime}}=\boldsymbol{Z}_{1}^{\prime} \mathbf{C W} \boldsymbol{Z}_{1}, \quad \frac{\partial^{2} L}{\partial \boldsymbol{\beta}_{2} \boldsymbol{\beta}_{2}^{\prime}}=\boldsymbol{Z}_{2}^{\prime} \mathbf{D T} \boldsymbol{Z}_{2} \quad \text { e } \frac{\partial^{2} L}{\partial \boldsymbol{\beta}_{1} \boldsymbol{\beta}_{2}^{\prime}}=\boldsymbol{Z}_{1}^{\prime} \mathbf{E W T} \boldsymbol{Z}_{2},
$$

com

$$
\begin{aligned}
& \mathbf{C}=\operatorname{diag}\left(c_{i j k m n}\right), \\
& c_{i j k m n}=\left(-\sum_{v=0}^{X_{i j k m n}-1} \frac{1}{\left(\mu_{j k m n}+v \theta_{j k m n}\right)^{2}}-\sum_{w=0}^{N_{i j k m n}-X_{i j k m n}-1} \frac{1}{\left(1-\mu_{j k m n}+w \theta_{j k m n}\right)^{2}}\right) \\
& \mu_{j k m n}\left(1-\mu_{j k m n}\right)+a_{i j k m n}\left(1-2 \mu_{j k m n}\right)
\end{aligned}
$$




$$
\begin{aligned}
& \mathbf{D}=\operatorname{diag}\left(d_{i j k m n}\right), \\
& d_{i j k m n}=\theta_{j k m n}\left[-\sum_{v=0}^{X_{i j k m n}-1}\left(\frac{v}{\mu_{j k m n}+v \theta_{j k m n}}\right)^{2}\right. \\
& \left.-\sum_{w=0}^{N_{i j k m n}-X_{i j k m n}-1}\left(\frac{w}{1-\mu_{j k m n}+w \theta_{j k m n}}\right)^{2}+\sum_{u=0}^{N_{i j k m n}-1}\left(\frac{u}{1+u \theta_{j k m n}}\right)^{2}\right]+b_{i j k m n} \\
& \mathbf{E}=\operatorname{diag}\left(e_{i j k m n}\right), \\
& e_{i j k m n}=-\sum_{v=0}^{X_{i j k m n}-1} \frac{v}{\left(\mu_{j k m n}+v \theta_{j k m n}\right)^{2}}+\sum_{w=0}^{N_{i j k m n}-X_{i j k m n}-1} \frac{w}{\left(1-\mu_{j k m n}+w \theta_{j k m n}\right)^{2}}
\end{aligned}
$$

e $\mathbf{Z}_{1}, \mathbf{Z}_{2}, \mathbf{W}$ e $\mathbf{T}$ definidos como anteriormente.

Detalhes das derivadas encontram-se no Apêndice B.

Como as soluções das primeiras derivadas não admitem forma fechada, para a maximização é necessário usar um processo iterativo como o processo de Newton-Raphson (ver, por exemplo, Thisted, 1988), que neste caso corresponde a iterar:

$$
\left(\begin{array}{c}
\boldsymbol{\beta}_{1} \\
\boldsymbol{\beta}_{2}
\end{array}\right)^{(m+1)}=\left(\begin{array}{c}
\boldsymbol{\beta}_{1} \\
\boldsymbol{\beta}_{2}
\end{array}\right)^{(m)}-\left[\left(\begin{array}{cc}
\boldsymbol{Z}_{1}^{\prime} \mathbf{C W} \boldsymbol{Z}_{1} & \boldsymbol{Z}_{1}^{\prime} \mathbf{E W T} \boldsymbol{Z}_{2} \\
\boldsymbol{Z}_{2}^{\prime} \mathbf{E W T} \boldsymbol{Z}_{1} & \boldsymbol{Z}_{2}^{\prime} \mathbf{D T} \boldsymbol{Z}_{2}
\end{array}\right)^{-1}\left(\begin{array}{c}
\boldsymbol{Z}_{1}^{\prime} \mathbf{W A} \\
\boldsymbol{Z}_{2}^{\prime} \mathbf{T B}
\end{array}\right)^{(m)}, m=0,1, \ldots\right.
$$

até que $\max \left|\beta_{s r}^{(m+1)}-\beta_{s r}^{(m)}\right|<0,00001\left(\mathrm{~s}=1,2 ; \mathrm{r}=1, \ldots, R_{s}\right)$, por exemplo.

Para os valores iniciais de $\mu_{j k m n}$ e $\theta_{j k m n}$, Griffiths (1973) sugere que se use o método dos momentos, com base na distribuição Beta-Binomial (2.6). Para os dados do estudo, esse método gerou valores iniciais negativos para alguns $\theta_{j k m n}$. Como alternativa foi usado o método dos momentos baseado na distribuição Beta (2.2), ou seja, considerando (2.4) e (2.5), que produzem os seguintes valores inicias:

$$
\begin{gathered}
\widetilde{\mu}_{j k m n}=\bar{\pi}_{j k m n} \\
\widetilde{\theta}_{j k m n}=\frac{s_{j k m n}^{2}}{\widetilde{\mu}_{j k m n}\left(1-\widetilde{\mu}_{j k m n}\right)-s_{j k m n}^{2}}
\end{gathered}
$$

com $\bar{\pi}_{j k m n}$ e $s_{j k m n}^{2}$ denotando, respectivamente, a média e a variância amostrais da proporção de acertos de indivíduos do grupo $j$, usando a mão $k$, na avaliação $m$ e seqüência 
n. Para gerar os valores iniciais para os parâmetros $\beta_{s r}\left(\mathrm{~s}=1,2 ; \mathrm{r}=1, \ldots, R_{s}\right)$, basta usar as funções de ligação dadas em (2.10) e (2.11) com os valores iniciais de $\mu_{j k m n}$ e $\theta_{j k m n}$.

O processo iterativo foi implementado no pacote S-Plus (ver, por exemplo, Everitt, 1994); o código correspondente encontra-se no endereço http://www.ime.usp.br/ mayra e os detalhes de como usá-lo, no Apêndice C. Para validá-lo, foram geradas amostras de distribuições Beta-Binomial, de diferentes tamanhos, confrontando-se os resultados gerados pelo algoritmo com os verdadeiros valores. Os resultados desta simulação estão apresentados no Apêndice D e mostram uma pequena variação dos valores estimados em relação ao original, especialmente considerando a média obtida em 5 simulações (a menor variação absoluta foi 0,5\% e a maior, 13\%). Nota-se também que quanto maior o tamanho da amostra, menor a variação e que os parâmetros relacionados a dispersão $\left(\boldsymbol{\beta}_{2}\right)$ variam mais do que os relacionados aos valores esperados da probabilidade de acerto $\left(\boldsymbol{\beta}_{1}\right)$.

Para selecionar as covariáveis significantes para o modelo final, foram usados testes da razão de verossimilhanças. Por exemplo, para verificar se o vetor de q parâmetros $\boldsymbol{\beta}_{2}^{*}\left(q<R_{2}\right)$ é nulo, através da hipótese $H_{0}: \boldsymbol{\beta}_{2}^{*}=0$, usamos a estatística de teste $R V=2\left(L^{*}-L\right)$, com $L^{*}$ denotando o logaritmo da verossimilhança sob $H_{0}$ e $\mathrm{L}$, esse logaritmo sob a hipótese alternativa. Assintoticamente, $R V$ segue uma distribuição $\chi_{q}^{2}$, sob a hipótese nula.

\subsection{Estimação sob o modelo Poisson Tetravariado}

Para as contagens totais das duas seqüências, nas duas avaliações, considerando o modelo (2.3), explicitamente temos

$$
\begin{aligned}
& P\left(N_{i j k I S}=n_{i j k I S}, N_{i j k I C}=n_{i j k I C}, N_{i j k F S}=n_{i j k F S}, N_{i j k F C}=n_{i j k F C}\right)= \\
& =\exp \left(-\lambda_{j k I S}-\lambda_{j k I C}-\lambda_{j k F S}-\lambda_{j k F C}+3 \lambda_{j k}\right) \\
& \sum_{u=0}^{\min \left(n_{i j k I S}, n_{i j k I C}, n_{i j k F S}, n_{i j k F C}\right)} \begin{array}{l}
{\left[\frac{\lambda_{j k}^{u}}{u !} \frac{\left(\lambda_{j k I S}-\lambda_{j k}\right)^{n_{i j k I S}-u}}{\left(n_{i j k I S}-u\right) !} \frac{\left(\lambda_{j k I C}-\lambda_{j k}\right)^{n_{i j k I C}-u}}{\left(n_{i j k I C}-u\right) !}\right.} \\
\left.\frac{\left(\lambda_{j k F S}-\lambda_{j k}\right)^{n_{i j k F S}-u}}{\left(n_{i j k F S}-u\right) !} \frac{\left(\lambda_{j k F C}-\lambda_{j k}\right)^{n_{i j k F C}-u}}{\left(n_{i j k F C}-u\right) !}\right]
\end{array}
\end{aligned}
$$

Da expressão $(2.12)$, segue que $\lambda_{j k}<\min \left(\lambda_{j k I S}, \lambda_{j k I C}, \lambda_{j k F S}, \lambda_{j k F C}\right)$. 
Com o objetivo de avaliar os efeitos dos fatores de interesse (estágio da doença e uso da mão preferencial), os parâmetros $\lambda_{j k I S}, \lambda_{j k I C}, \lambda_{j k F S}, \lambda_{j k F C}$ e $\lambda_{j k}$ podem ser modelados por

$$
\begin{aligned}
& \lambda_{j k m n}=\exp \left(\mathbf{z}_{s i j k}^{\prime} \boldsymbol{\beta}_{s}\right), \quad s=1, \ldots, 4 \\
& \lambda_{j k}=\exp \left(\mathbf{z}_{5 i j k}^{\prime} \boldsymbol{\beta}_{5}\right)
\end{aligned}
$$

ou equivalentemente, por

$$
\begin{aligned}
& \eta_{s}=\log \left(\lambda_{j k m n}\right)=\mathbf{z}_{s i j k}^{\prime} \boldsymbol{\beta}_{s}, \quad s=1, \ldots, 4 \\
& \eta_{5}=\log \left(\lambda_{j k}\right)=\mathbf{z}_{5 i j k}^{\prime} \boldsymbol{\beta}_{5}
\end{aligned}
$$

em que $\mathbf{z}_{s i j k}(\mathrm{~s}=1, \ldots, 5)$ são vetores de variáveis explicativas associados a cada indivíduo e $\boldsymbol{\beta}_{s}(\mathrm{~s}=1, \ldots, 5)$ são vetores de parâmetros.

Para estimar os parâmetros de $\boldsymbol{\beta}_{s}(\mathrm{~s}=1, \ldots, 5)$ via máxima verossimilhança consideramos as primeiras derivadas da função log-verossimilhança, que em notação matricial podem ser expressas como

$$
\frac{\partial L}{\partial \boldsymbol{\beta}_{1}}=\boldsymbol{Z}_{1}^{\prime} \boldsymbol{L}_{I S} \mathbf{A}, \frac{\partial L}{\partial \boldsymbol{\beta}_{2}}=\boldsymbol{Z}_{2}^{\prime} \boldsymbol{L}_{I C} \mathbf{B}, \frac{\partial L}{\partial \boldsymbol{\beta}_{3}}=\boldsymbol{Z}_{3}^{\prime} \boldsymbol{L}_{F S} \mathbf{C}, \frac{\partial L}{\partial \boldsymbol{\beta}_{4}}=\boldsymbol{Z}_{4}^{\prime} \boldsymbol{L}_{F C} \mathbf{D} \text { e } \frac{\partial L}{\partial \boldsymbol{\beta}_{5}}=\boldsymbol{Z}_{5}^{\prime} \mathbf{L E}
$$

com

$$
\begin{aligned}
& \mathbf{A}=\left(a_{10 k}, \ldots, a_{i j k}, \ldots, a_{12,2 k}\right)^{\prime}, \quad a_{i j k}=P_{1000}-1 \\
& \mathbf{B}=\left(b_{10 k}, \ldots, b_{i j k}, \ldots, b_{12,2 k}\right)^{\prime}, \quad b_{i j k}=P_{0100}-1 \\
& \mathbf{C}=\left(c_{10 k}, \ldots, c_{i j k}, \ldots, c_{12,2 k}\right)^{\prime}, \quad c_{i j k}=P_{0010}-1 \\
& \mathbf{D}=\left(d_{10 k}, \ldots, d_{i j k}, \ldots, d_{12,2 k}\right)^{\prime}, \quad d_{i j k}=P_{0001}-1 \\
& \mathbf{E}=\left(e_{10 k}, \ldots, e_{i j k}, \ldots, e_{12,2 k}\right)^{\prime}, e_{i j k}=3+P_{1111}-P_{1000}-P_{0100}-P_{0010}-P_{0001} \\
& \mathbf{Z}_{s}=\left(\mathbf{z}_{s 10 k}^{\prime}, \ldots, \mathbf{z}_{s i j k}^{\prime}, \ldots, \mathbf{z}_{s, 12,2 k}^{\prime}\right), s=1, \ldots, 5 \\
& \boldsymbol{L}_{I S}=\operatorname{diag}\left(\lambda_{j k I S}\right), \boldsymbol{L}_{I C}=\operatorname{diag}\left(\lambda_{j k I C}\right), \boldsymbol{L}_{F S}=\operatorname{diag}\left(\lambda_{j k F S}\right), \boldsymbol{L}_{F C}=\operatorname{diag}\left(\lambda_{j k F C}\right) \mathrm{e} \\
& \mathbf{L}=\operatorname{diag}\left(\lambda_{j k}\right)
\end{aligned}
$$

em que $P_{u v x y}=$

$$
\frac{P\left(N_{i j k I S}=n_{i j k I S}-u, N_{i j k I C}=n_{i j k I C}-v, N_{i j k F S}=n_{i j k F S}-x, N_{i j k F C}=n_{i j k F C}-y\right)}{P\left(N_{i j k I S}=n_{i j k I S}, N_{i j k I C}=n_{i j k I C}, N_{i j k F S}=n_{i j k F S}, N_{i j k F C}=n_{i j k F C}\right)}
$$


usando a notação proposta por Kocherlakota e Kocherlakota (2001). As segundas derivadas podem ser expressas como

$$
\begin{gathered}
\frac{\partial^{2} L}{\partial \boldsymbol{\beta}_{1} \boldsymbol{\beta}_{1}^{\prime}}=\boldsymbol{Z}_{1}^{\prime} \mathbf{F} \boldsymbol{L}_{I S} \boldsymbol{Z}_{1}, \frac{\partial^{2} L}{\partial \boldsymbol{\beta}_{1} \boldsymbol{\beta}_{2}^{\prime}}=\boldsymbol{Z}_{1}^{\prime} \mathbf{G} \boldsymbol{L}_{I S} \boldsymbol{L}_{I C} \boldsymbol{Z}_{2}, \frac{\partial^{2} L}{\partial \boldsymbol{\beta}_{1} \boldsymbol{\beta}_{3}^{\prime}}=\boldsymbol{Z}_{1}^{\prime} \mathbf{H} \boldsymbol{L}_{I S} \boldsymbol{L}_{F S} \boldsymbol{Z}_{3} \\
\frac{\partial^{2} L}{\partial \boldsymbol{\beta}_{1} \boldsymbol{\beta}_{4}^{\prime}}=\boldsymbol{Z}_{1}^{\prime} \mathbf{M} \boldsymbol{L}_{I S} \boldsymbol{L}_{F C} \boldsymbol{Z}_{4}, \frac{\partial^{2} L}{\partial \boldsymbol{\beta}_{1} \boldsymbol{\beta}_{5}^{\prime}}=\boldsymbol{Z}_{1}^{\prime} \mathbf{N} \boldsymbol{L}_{I S} \mathbf{L} \boldsymbol{Z}_{5}, \frac{\partial^{2} L}{\partial \boldsymbol{\beta}_{2} \boldsymbol{\beta}_{2}^{\prime}}=\boldsymbol{Z}_{2}^{\prime} \mathbf{P} \boldsymbol{L}_{I C} \boldsymbol{Z}_{2}, \\
\frac{\partial^{2} L}{\partial \boldsymbol{\beta}_{2} \boldsymbol{\beta}_{3}^{\prime}}=\boldsymbol{Z}_{2}^{\prime} \mathbf{Q} \boldsymbol{L}_{I C} \boldsymbol{L}_{F S} \boldsymbol{Z}_{3}, \frac{\partial^{2} L}{\partial \boldsymbol{\beta}_{2} \boldsymbol{\beta}_{4}^{\prime}}=\boldsymbol{Z}_{2}^{\prime} \mathbf{R} \boldsymbol{L}_{I C} \boldsymbol{L}_{F C} \boldsymbol{Z}_{4}, \frac{\partial^{2} L}{\partial \boldsymbol{\beta}_{2} \boldsymbol{\beta}_{5}^{\prime}}=\boldsymbol{Z}_{2}^{\prime} \mathbf{S} \boldsymbol{L}_{I C} \mathbf{L} \boldsymbol{Z}_{5}, \\
\frac{\partial^{2} L}{\partial \boldsymbol{\beta}_{3} \boldsymbol{\beta}_{3}^{\prime}}=\boldsymbol{Z}_{3}^{\prime} \mathbf{T} \boldsymbol{L}_{F S} \boldsymbol{Z}_{3}, \frac{\partial^{2} L}{\partial \boldsymbol{\beta}_{3} \boldsymbol{\beta}_{4}^{\prime}}=\boldsymbol{Z}_{3}^{\prime} \mathbf{U} \boldsymbol{L}_{F S} \boldsymbol{L}_{F C} \boldsymbol{Z}_{4}, \frac{\partial^{2} L}{\partial \boldsymbol{\beta}_{3} \boldsymbol{\beta}_{5}^{\prime}}=\boldsymbol{Z}_{3}^{\prime} \mathbf{V} \boldsymbol{L}_{F S} \mathbf{L} \boldsymbol{Z}_{5}, \\
\frac{\partial^{2} L}{\partial \boldsymbol{\beta}_{4} \boldsymbol{\beta}_{4}^{\prime}}=\boldsymbol{Z}_{4}^{\prime} \mathbf{W} \boldsymbol{L}_{F C} \boldsymbol{Z}_{4}, \frac{\partial^{2} L}{\partial \boldsymbol{\beta}_{4} \boldsymbol{\beta}_{5}^{\prime}}=\boldsymbol{Z}_{4}^{\prime} \mathbf{X} \boldsymbol{L}_{F C} \mathbf{L} \boldsymbol{Z}_{5} \quad \text { e } \frac{\partial^{2} L}{\partial \boldsymbol{\beta}_{5} \boldsymbol{\beta}_{5}^{\prime}}=\boldsymbol{Z}_{5}^{\prime} \mathbf{Y L} \boldsymbol{Z}_{5},
\end{gathered}
$$

com

$$
\begin{aligned}
& \mathbf{F}=\operatorname{diag}\left(f_{i j k}\right), \quad f_{i j k}=P_{1000}-1+\lambda_{j k I S}\left(P_{2000}-P_{1000}^{2}\right) \\
& \mathbf{G}=\operatorname{diag}\left(g_{i j k}\right), \quad g_{i j k}=P_{1100}-P_{1000} P_{0100} \\
& \mathbf{H}=\operatorname{diag}\left(h_{i j k}\right), \quad h_{i j k}=P_{1010}-P_{1000} P_{0010} \\
& \mathbf{M}=\operatorname{diag}\left(m_{i j k}\right), \quad m_{i j k}=P_{1001}-P_{1000} P_{0001} \\
& \mathbf{N}=\operatorname{diag}\left(n_{i j k}\right), \quad n_{i j k}=P_{2111}-P_{2000}-P_{1100}-P_{1010}-P_{1001} \\
& +P_{1000}\left(P_{1000}+P_{0100}+P_{0010}+P_{0001}-P_{1111}\right) \\
& \mathbf{P}=\operatorname{diag}\left(p_{i j k}\right), \quad p_{i j k}=P_{0100}-1+\lambda_{j k I C}\left(P_{0200}-P_{0100}^{2}\right) \\
& \mathbf{Q}=\operatorname{diag}\left(q_{i j k}\right), \quad q_{i j k}=P_{0110}-P_{0100} P_{0010} \\
& \mathbf{R}=\operatorname{diag}\left(r_{i j k}\right), \quad r_{i j k}=P_{0101}-P_{0100} P_{0001} \\
& \mathbf{S}=\operatorname{diag}\left(s_{i j k}\right), \quad s_{i j k}=P_{1211}-P_{1100}-P_{0200}-P_{0110}-P_{0101} \\
& +P_{0100}\left(P_{1000}+P_{0100}+P_{0010}+P_{0001}-P_{1111}\right) \\
& \mathbf{T}=\operatorname{diag}\left(t_{i j k}\right), \quad t_{i j k}=P_{0010}-1+\lambda_{j k F S}\left(P_{0020}-P_{0010}^{2}\right) \\
& \mathbf{U}=\operatorname{diag}\left(u_{i j k}\right), \quad u_{i j k}=P_{0011}-P_{0010} P_{0001} \\
& \mathbf{V}=\operatorname{diag}\left(v_{i j k}\right), \quad v_{i j k}=P_{1121}-P_{1010}-P_{0110}-P_{0020}-P_{0011} \\
& +P_{0010}\left(P_{1000}+P_{0100}+P_{0010}+P_{0001}-P_{1111}\right)
\end{aligned}
$$




$$
\begin{aligned}
& \mathbf{W}=\operatorname{diag}\left(w_{i j k}\right), \quad w_{i j k}=P_{0001}-1+\lambda_{j k F C}\left(P_{0002}-P_{0001}^{2}\right) \\
& \mathbf{X}=\operatorname{diag}\left(x_{i j k}\right), \quad x_{i j k}=P_{1112}-P_{1001}-P_{0101}-P_{0011}-P_{0002} \\
& +P_{0001}\left(P_{1000}+P_{0100}+P_{0010}+P_{0001}-P_{1111}\right) \\
& \mathbf{Y}=\operatorname{diag}\left(y_{i j k}\right), y_{i j k}=e_{i j k}+\lambda_{j k}\left(P_{2222}-P_{2111}-P_{1211}-P_{1121}-P_{1112}\right. \\
& \left.+P_{1111}\left(P_{1000}+P_{0100}+P_{0010}+P_{0001}-P_{1111}\right)-n_{i j k}-s_{i j k}-v_{i j k}-x_{i j k}\right) \\
& \text { e } P_{u v x y}, \quad \mathbf{Z}_{s}, \quad s=1, \ldots, 5, \quad \boldsymbol{L}_{I S}, \quad \boldsymbol{L}_{I C}, \quad \boldsymbol{L}_{F S}, \quad \boldsymbol{L}_{F C} \text { e } \mathbf{L} \text { definidos como anteriormente. }
\end{aligned}
$$

Deve-se destacar que quando $n_{i j k m n}=1$, a segunda derivada é igual a zero. Mais detalhes sobre essas derivadas encontram-se no Apêndice B.

Para a maximização da log-verossimilhança é necessário usar um processo iterativo como o processo de Newton-Raphson, que neste caso corresponde a iterar:

$$
\left(\begin{array}{c}
\boldsymbol{\beta}_{1} \\
\boldsymbol{\beta}_{2} \\
\boldsymbol{\beta}_{3} \\
\boldsymbol{\beta}_{4} \\
\boldsymbol{\beta}_{5}
\end{array}\right)^{(m+1)}=\left(\begin{array}{c}
\boldsymbol{\beta}_{1} \\
\boldsymbol{\beta}_{2} \\
\boldsymbol{\beta}_{3} \\
\boldsymbol{\beta}_{4} \\
\boldsymbol{\beta}_{5}
\end{array}\right)^{(m)}-\left[\mathbf{K}^{(m)}\right]^{-1}\left(\begin{array}{c}
\boldsymbol{Z}_{1}^{\prime} \boldsymbol{L}_{I S} \mathbf{A} \\
\boldsymbol{Z}_{2}^{\prime} \boldsymbol{L}_{I C} \mathbf{B} \\
\boldsymbol{Z}_{3}^{\prime} \boldsymbol{L}_{F S} \mathbf{C} \\
\boldsymbol{Z}_{4}^{\prime} \boldsymbol{L}_{F C} \mathbf{D} \\
\boldsymbol{Z}_{5}^{\prime} \mathbf{L E}
\end{array}\right)^{(m)}, m=0,1, \ldots
$$

$\boldsymbol{K}=\left(\begin{array}{ll}\boldsymbol{K}_{1} & \boldsymbol{K}_{2}\end{array}\right), \mathrm{com}$

$$
\begin{aligned}
\boldsymbol{K}_{1}= & \left(\begin{array}{ccc}
\boldsymbol{Z}_{1}^{\prime} \mathbf{F} \boldsymbol{L}_{I S} \boldsymbol{Z}_{1} & \boldsymbol{Z}_{1}^{\prime} \mathrm{G} \boldsymbol{L}_{I S} \boldsymbol{L}_{I C} \boldsymbol{Z}_{2} & \boldsymbol{Z}_{1}^{\prime} \mathbf{H} \boldsymbol{L}_{I S} \boldsymbol{L}_{F S} \boldsymbol{Z}_{3} \\
\boldsymbol{Z}_{2}^{\prime} \mathrm{G} \boldsymbol{L}_{I S} \boldsymbol{L}_{I C} \boldsymbol{Z}_{1} & \boldsymbol{Z}_{2}^{\prime} \mathbf{P} \boldsymbol{L}_{I C} \boldsymbol{Z}_{2} & \boldsymbol{Z}_{2}^{\prime} \mathrm{Q} \boldsymbol{L}_{I C} \boldsymbol{L}_{F S} \boldsymbol{Z}_{3} \\
\boldsymbol{Z}_{3}^{\prime} \mathbf{H} \boldsymbol{L}_{I S} \boldsymbol{L}_{F S} \boldsymbol{Z}_{1} & \boldsymbol{Z}_{3}^{\prime} \mathrm{Q} \boldsymbol{L}_{I C} \boldsymbol{L}_{F S} \boldsymbol{Z}_{2} & \boldsymbol{Z}_{3}^{\prime} \mathbf{T} \boldsymbol{L}_{F S} \boldsymbol{Z}_{3} \\
\boldsymbol{Z}_{4}^{\prime} \mathrm{M} \boldsymbol{L}_{I S} \boldsymbol{L}_{F C} \boldsymbol{Z}_{1} & \boldsymbol{Z}_{4}^{\prime} \mathbf{R} \boldsymbol{L}_{I C} \boldsymbol{L}_{F C} \boldsymbol{Z}_{2} & \boldsymbol{Z}_{4}^{\prime} \mathrm{U} \boldsymbol{L}_{F S} \boldsymbol{L}_{F C} \boldsymbol{Z}_{3} \\
\boldsymbol{Z}_{5}^{\prime} \mathrm{N} \boldsymbol{L}_{I S} \mathbf{L} \boldsymbol{Z}_{1} & \boldsymbol{Z}_{5}^{\prime} \mathrm{S} \boldsymbol{L}_{I C} \mathbf{L} \boldsymbol{Z}_{2} & \boldsymbol{Z}_{5}^{\prime} \mathbf{V} \boldsymbol{L}_{F S} \mathbf{L} \boldsymbol{Z}_{3}
\end{array}\right) \\
\boldsymbol{K}_{2}= & \left(\begin{array}{cc}
\boldsymbol{Z}_{1}^{\prime} \mathrm{M} \boldsymbol{L}_{I S} \boldsymbol{L}_{F C} \boldsymbol{Z}_{4} & \boldsymbol{Z}_{1}^{\prime} \mathbf{N} \boldsymbol{L}_{I S} \mathbf{L} \boldsymbol{Z}_{5} \\
\boldsymbol{Z}_{2}^{\prime} \mathbf{R} \boldsymbol{L}_{I C} \boldsymbol{L}_{F C} \boldsymbol{Z}_{4} & \boldsymbol{Z}_{2}^{\prime} \mathrm{S} \boldsymbol{L}_{I C} \mathbf{L} \boldsymbol{Z}_{5} \\
\boldsymbol{Z}_{3}^{\prime} \mathrm{U} \boldsymbol{L}_{F S} \boldsymbol{L}_{F C} \boldsymbol{Z}_{4} & \boldsymbol{Z}_{3}^{\prime} \mathbf{V} \boldsymbol{L}_{F S} \mathbf{L} \boldsymbol{Z}_{5} \\
\boldsymbol{Z}_{4}^{\prime} \mathbf{W} \boldsymbol{L}_{F C} \boldsymbol{Z}_{4} & \boldsymbol{Z}_{4}^{\prime} \mathbf{X} \boldsymbol{L}_{F C} \mathbf{L} \boldsymbol{Z}_{5} \\
\boldsymbol{Z}_{5}^{\prime} \mathrm{X} \boldsymbol{L}_{F C} \mathbf{L} \boldsymbol{Z}_{4} & \boldsymbol{Z}_{5}^{\prime} \mathbf{Y} \mathbf{L} \boldsymbol{Z}_{5}
\end{array}\right)
\end{aligned}
$$

até que $\max \left|\beta_{s r}^{(m+1)}-\beta_{s r}^{(m)}\right|<0,00001\left(\mathrm{~s}=1, \ldots, 5 ; \mathrm{r}=1, \ldots, R_{s}\right)$, por exemplo.

Para os valores iniciais de $\lambda_{j k I S}, \lambda_{j k I C}, \lambda_{j k F S}, \lambda_{j k F C}$ e $\lambda_{j k}$, o método dos momentos 
pode ser utilizado, de maneira que

$$
\begin{gathered}
\lambda_{j k m n}^{(0)}=\bar{n}_{j k m n}, m=I, F, n=S, C \\
\lambda_{j k}^{(0)}=s_{j k m n}
\end{gathered}
$$

$\operatorname{com} \bar{n}_{j k m n}$ denotando a média amostral do total de tentativas de indivíduos do grupo $j$, usando a mão $k$, na avaliação $m$ e seqüência $n$ e $s_{j k m n}$ a covariância amostral entre duas contagens de totais ou a menor média amostral observada, já que $\lambda_{j k}<\min \left(\lambda_{j k I S}, \lambda_{j k I C}\right.$, $\left.\lambda_{j k F S}, \lambda_{j k F C}\right)$. Para gerar os valores iniciais para os parâmetros $\beta_{s r}\left(\mathrm{~s}=1, \ldots, 5 ; \mathrm{r}=1, \ldots, R_{s}\right)$, basta usar (2.13) e (2.14) com os valores iniciais de $\lambda_{j k m n}$ e $\lambda_{j k}$.

O processo iterativo foi implementado no pacote S-Plus; o código correspondente encontra-se no endereço http://www.ime.usp.br/ mayra e os detalhes de como usá-lo, no Apêndice C. Para validá-lo, foram geradas amostras de distribuições Poisson Tetravariada, de diferentes tamanhos, confrontando-se os resultados gerados pelo algoritmo com os verdadeiros valores. Os resultados desta simulação estão apresentados no Apêndice D e mostram uma pequena variação dos valores estimados em relação ao original, especialmente considerando a média obtida em 5 simulações (sendo observados casos sem variação). Nota-se também que quanto maior o tamanho da amostra, menor a variação e que os parâmetros relacionados a covariância $\left(\boldsymbol{\beta}_{5}\right)$ variam mais do que os relacionados aos valores esperados do total de tentativas $\left(\boldsymbol{\beta}_{s}, \mathrm{~s}=1, \ldots, 4\right)$, ocorrendo dois casos com variações absolutas médias maiores que $40 \%$.

Para selecionar as covariáveis significantes para o modelo final, foram usados testes da razão de verossimilhanças similiares àqueles apresentados para a estimação sob o modelo Beta-Binomial. 


\section{Capítulo 3}

\section{Análise dos dados}

Neste capítulo, analisamos os dados do estudo descrito no Capítulo 1. Na seção 3.1 serão apresentados os resultados obtidos para as componentes modeladas pela distribuição Beta-Binomial, na seção 3.2, os resultados para o modelo Poisson Tetravariado e, por fim, na seção 3.3, os resultados finais, gerando os valores esperados para as contagens de acertos e totais.

\subsection{Modelagem do número de acertos}

Para verificar o efeito dos fatores de interesse na probabilidade de acerto, inicialmente foi ajustado um modelo com todos os efeitos principais e interações de primeira ordem. O método iterativo proposto mostrou-se muito sensível aos valores iniciais, especialmente para as interações. Por isso, inicialmente foi ajustado um modelo com apenas os efeitos principais; as estimativas dos parâmetros desse modelo foram usadas como valores iniciais para o ajuste de outros modelos obtidos com a inclusão das interações, uma a uma, no modelo. O valor inicial de cada interação foi dado pelo método dos momentos proposto ou, quando o processo iterativo não convergia, tal valor era substituído por zero. Por fim, as estimativas de cada interação (ou no caso das que não haviam convergido, zero) foram usadas como valores iniciais para o modelo completo.

Após o ajuste desse modelo completo, testaram-se através da razão de verossimi- 
lhanças, hipóteses do tipo

$$
H_{0}: \beta_{s r}=0
$$

para verificar quais parâmetros eram significantes; iniciamos o processo com as interações e, em seguida, consideramos os efeitos principais.

Para comparar os grupos de estudo com relação às duas avaliações e às seqüências treinada e controle, inicialmente foi ajustado o modelo saturado com parametrização de casela de referência, em que os vetores de parâmetros são

$$
\begin{aligned}
\boldsymbol{\beta}_{s}= & \left(\beta_{s 0}, \beta_{s 1}, \beta_{s 2}, \beta_{s M}, \beta_{s F}, \beta_{s C},\right. \\
& \left.\beta_{s(1 * F)}, \beta_{s(1 * M)}, \beta_{s(1 * C)}, \beta_{s(2 * F)}, \beta_{s(2 * M)}, \beta_{s(2 * C)}, \beta_{s(F * M)}, \beta_{s(F * C)}, \beta_{s(M * C)}\right), \quad s=1,2 .
\end{aligned}
$$

Neste contexto,

- $\beta_{10}$ é o logaritmo da razão entre os valores esperados da probabilidade de acerto e de erro de um indivíduo do grupo controle, usando a mão preferencial, na avaliação inicial, executado a seqüência treinada;

- $\beta_{11}$ é a diferença entre os logaritmos das razões entre os valores esperados da probabilidade de acerto e de erro de indivíduos em estágio inicial da doença e controles;

- $\beta_{12}$ é a diferença entre os logaritmos das razões entre os valores esperados da probabilidade de acerto e de erro de indivíduos em estágio avançado da doença e controles;

- $\beta_{1 M}$ é a diferença entre os logaritmos das razões entre os valores esperados da probabilidade de acerto e de erro de indivíduos usando a mão não preferencial e a preferencial;

- $\beta_{1 F}$ é a diferença entre os logaritmos das razões entre os valores esperados da probabilidade de acerto e de erro na avaliação final e inicial;

- $\beta_{1 C}$ é a diferença entre os logaritmos das razões entre os valores esperados da probabilidade de acerto e de erro da seqüência controle e da treinada.

- $\beta_{20}$ é o logaritmo do valor esperado do parâmetro relacionado à dispersão de um indivíduo do grupo controle, usando a mão preferencial, na avaliação inicial, executado a seqüência treinada; 
- $\beta_{21}$ é a diferença entre os logaritmos dos valores esperados dos parâmetros relacionados à dispersão de indivíduos em estágio inicial da doença e controles;

- $\beta_{22}$ é a diferença entre os logaritmos dos valores esperados dos parâmetros relacionados à dispersão de indivíduos em estágio avançado da doença e controles;

- $\beta_{2 M}$ é a diferença entre os logaritmos dos valores esperados dos parâmetros relacionados à dispersão de indivíduos usando a mão não preferencial e a preferencial;

- $\beta_{2 F}$ é a diferença entre os logaritmos dos valores esperados dos parâmetros relacionados à dispersão na avaliação final e inicial;

- $\beta_{2 C}$ é a diferença entre os logaritmos dos valores esperados dos parâmetros relacionados à dispersão da seqüência controle e da treinada.

Com o modelo ajustado, inicialmente verificou-se quais interações eram significantes através da hipótese

$$
\begin{array}{cc}
H_{0}: \quad \beta_{1(1 * F)}, \beta_{1(1 * M)}, \beta_{1(1 * C)}, \beta_{1(2 * F)}, \beta_{1(2 * M)}, \beta_{1(2 * C)}, \beta_{1(F * M)}, \beta_{1(M * C)}, \\
\beta_{2(1 * C)}, \beta_{2(2 * F)}, \beta_{2(2 * C)}, \beta_{2(2 * M)}, \beta_{2(F * C)}, \beta_{1(M * C)}=0
\end{array}
$$

obtendo-se $\mathrm{p}=0,543$, sendo então eliminados tais parâmetros e ajustado um modelo reduzido. A partir desse modelo, verificou-se quais efeitos principais eram significantes através da hipótese

$$
\begin{aligned}
H_{0}: & \beta_{11}, \beta_{1 M}, \beta_{1 C}, \\
& \beta_{2 M}, \beta_{2 C}, \beta_{2 F}=0
\end{aligned}
$$

obtendo-se $\mathrm{p}=0,210$, sendo também retirados esses parâmetros do modelo e então ajustado o modelo final apresentado na Tabela 3.1, já que outras hipóteses de que algum $\beta_{s r}$ fosse igual a zero foram rejeitadas, todas com $\mathrm{p}<0,150$.

A partir dessas estimativas pode-se calcular os valores esperados $\left(\mu_{j k m n}\right)$ para as probabilidades de acerto $\left(\pi_{i j k m n}\right)$ dos diferentes grupos de estudo (Tabela 3.2) e para os parâmetros $\theta_{j k m n}$ relacionados à variabilidade da probabilidade de acerto (Tabela 3.3).

Considerando os resultados apresentados nas Tabelas 3.1 e 3.2 para o valor esperado da probabilidade de acerto, pode-se concluir que: 
Tabela 3.1: Estimativas dos parâmetros sob o modelo Beta-Binomial final

\begin{tabular}{llcc}
\hline Parâmetro & Relacionado ao & Estimativa & Erro Padrão \\
\hline $\boldsymbol{\beta}_{1}$ & vetor de valores esperados da probabilidade de acerto & & \\
$\beta_{10}$ & grupo controle, mão preferencial, & 1,86 & 0,15 \\
& avaliação inicial e seqüência treinada & & \\
$\beta_{12}$ & efeito da doença em estágio avançado & $-1,35$ & 0,25 \\
$\beta_{1 F}$ & efeito da avaliação final & 1,38 & 0,30 \\
$\beta_{1(F * C)}$ & interação: avaliação final e seqüência controle & $-1,79$ & 0,30 \\
\hline $\boldsymbol{\beta}_{2}$ & vetor de valores esperados do parâmetro de dispersão & & \\
$\beta_{20}$ & grupo controle, mão preferencial, & $-1,07$ & 0,27 \\
& avaliação inicial e seqüência treinada & & \\
$\beta_{21}$ & efeito da doença em estágio inicial & $-2,98$ & 1,05 \\
$\beta_{22}$ & efeito da doença em estágio avançado & 1,31 & 0,37 \\
$\beta_{2(1 * F)}$ & interação: estágio inicial e avaliação final & 1,66 & 0,82 \\
$\beta_{2(1 * M)}$ & interação: estágio inicial e mão não preferencial & 2,78 & 0,91 \\
$\beta_{2(F * M)}$ & interação: avaliação final e mão não preferencial & $-1,49$ & 0,44 \\
\hline
\end{tabular}

- não há diferença entre as probabilidades com a mão preferencial e com a mão não preferencial $\left(\beta_{1 M}=0\right)$;

- indivíduos do grupo controle e de estágio inicial da doença têm igual probabilidade de acerto $\left(\beta_{11}=0\right)$;

- indivíduos no estágio avançado da doença têm menor probabilidade de acerto do que os controles $\left(\beta_{12}<0\right)$;

- não há diferença entre as probabilidades de acerto referentes às duas seqüências antes do treinamento $\left(\beta_{1 C}=0\right)$;

- há efeito do treinamento: a probabilidade de acertar a seqüência treinada na avaliação final aumenta relativamente à avaliação inicial $\left(\beta_{1 F}>0\right)$;

- a probabilidade de acerto da seqüência controle na avaliação final é menor do que na inicial $\left(\beta_{1 F}+\beta_{1(F * C)}<0\right)$. 
Tabela 3.2: Estimativas dos valores esperados das probabilidades de acertos para os grupos de estudo nas diferentes situações de realização das seqüências

\begin{tabular}{|c|c|c|c|c|c|c|}
\hline $\begin{array}{c}\text { Estágio } \\
\text { da doença }\end{array}$ & Avaliação & Mão & Seqüência & Parâmetros & $\begin{array}{c}\text { Valor } \\
\text { esperado }\end{array}$ & $\begin{array}{c}\text { Erro } \\
\text { padrão }\end{array}$ \\
\hline controle & inicial & preferencial & treinada & $\beta_{10}$ & 0,87 & 0,02 \\
\hline controle & inicial & preferencial & controle & $\beta_{10}$ & 0,87 & 0,02 \\
\hline controle & inicial & não & treinada & $\beta_{10}$ & 0,87 & 0,02 \\
\hline controle & inicial & não & controle & $\beta_{10}$ & 0,87 & 0,02 \\
\hline controle & final & preferencial & treinada & $\beta_{10}+\beta_{1 F}$ & 0,96 & 0,02 \\
\hline controle & final & preferencial & controle & $\beta_{10}+\beta_{1 F}+\beta_{1(F * C)}$ & 0,81 & 0,12 \\
\hline controle & final & não & treinada & $\beta_{10}+\beta_{1 F}$ & 0,96 & 0,02 \\
\hline controle & final & não & controle & $\beta_{10}+\beta_{1 F}+\beta_{1(F * C)}$ & 0,81 & 0,12 \\
\hline inicial & inicial & preferencial & treinada & $\beta_{10}$ & 0,87 & 0,02 \\
\hline inicial & inicial & preferencial & controle & $\beta_{10}$ & 0,87 & 0,02 \\
\hline inicial & inicial & não & treinada & $\beta_{10}$ & 0,87 & 0,02 \\
\hline inicial & inicial & não & controle & $\beta_{10}$ & 0,87 & 0,02 \\
\hline inicial & final & preferencial & treinada & $\beta_{10}+\beta_{1 F}$ & 0,96 & 0,02 \\
\hline inicial & final & preferencial & controle & $\beta_{10}+\beta_{1 F}+\beta_{1(F * C)}$ & 0,81 & 0,12 \\
\hline inicial & final & não & treinada & $\beta_{10}+\beta_{1 F}$ & 0,96 & 0,02 \\
\hline inicial & final & não & controle & $\beta_{10}+\beta_{1 F}+\beta_{1(F * C)}$ & 0,81 & 0,12 \\
\hline avançado & inicial & preferencial & treinada & $\beta_{10}+\beta_{12}$ & 0,62 & 0,09 \\
\hline avançado & inicial & preferencial & controle & $\beta_{10}+\beta_{12}$ & 0,62 & 0,09 \\
\hline avançado & inicial & não & treinada & $\beta_{10}+\beta_{12}$ & 0,62 & 0,09 \\
\hline avançado & inicial & não & controle & $\beta_{10}+\beta_{12}$ & 0,62 & 0,09 \\
\hline avançado & final & preferencial & treinada & $\beta_{10}+\beta_{12}+\beta_{1 F}$ & 0,88 & 0,08 \\
\hline avançado & final & preferencial & controle & $\beta_{10}+\beta_{12}+\beta_{1 F}+\beta_{1(F * C)}$ & 0,52 & 0,24 \\
\hline avançado & final & não & treinada & $\beta_{10}+\beta_{12}+\beta_{1 F}$ & 0,88 & 0,08 \\
\hline avançado & final & não & controle & $\beta_{10}+\beta_{12}+\beta_{1 F}+\beta_{1(F * C)}$ & 0,52 & 0,24 \\
\hline
\end{tabular}


Tabela 3.3: Estimativas dos parâmetros relacionados à dispersão $\theta_{j k m n}$ para os grupos de estudo nas diferentes situações de realização das seqüências

\begin{tabular}{|c|c|c|c|c|c|c|}
\hline $\begin{array}{c}\text { Estágio } \\
\text { da doença }\end{array}$ & Avaliação & Mão & Seqüência & Parâmetros & $\begin{array}{c}\text { Valor } \\
\text { esperado }\end{array}$ & $\begin{array}{c}\text { Erro } \\
\text { padrão }\end{array}$ \\
\hline controle & inicial & preferencial & treinada & $\beta_{20}$ & 0,34 & 0,09 \\
\hline controle & inicial & preferencial & controle & $\beta_{20}$ & 0,34 & 0,09 \\
\hline controle & inicial & não & treinada & $\beta_{20}$ & 0,34 & 0,09 \\
\hline controle & inicial & não & controle & $\beta_{20}$ & 0,34 & 0,09 \\
\hline controle & final & preferencial & treinada & $\beta_{20}$ & 0,34 & 0,09 \\
\hline controle & final & preferencial & controle & $\beta_{20}$ & 0,34 & 0,09 \\
\hline controle & final & não & treinada & $\beta_{20}+\beta_{2(F * M)}$ & 0,08 & 0,06 \\
\hline controle & final & não & controle & $\beta_{20}+\beta_{2(F * M)}$ & 0,08 & 0,06 \\
\hline inicial & inicial & preferencial & treinada & $\beta_{20}+\beta_{21}$ & 0,02 & 0,03 \\
\hline inicial & inicial & preferencial & controle & $\beta_{20}+\beta_{21}$ & 0,02 & 0,03 \\
\hline inicial & inicial & não & treinada & $\beta_{20}+\beta_{21}+\beta_{2(1 * M)}$ & 0,28 & 1,28 \\
\hline inicial & inicial & não & controle & $\beta_{20}+\beta_{21}+\beta_{2(1 * M)}$ & 0,28 & 1,28 \\
\hline inicial & final & preferencial & treinada & $\beta_{20}+\beta_{21}+\beta_{2(1 * F)}$ & 0,09 & 0,38 \\
\hline inicial & final & preferencial & controle & $\beta_{20}+\beta_{21}+\beta_{2(1 * F)}$ & 0,09 & 0,38 \\
\hline inicial & final & não & treinada & $\beta_{20}+\beta_{21}+\beta_{2(1 * F)}$ & 0,33 & 5,45 \\
\hline inicial & final & não & controle & $\begin{array}{l}+\beta_{2(1 * M)}+\beta_{2(F * M)} \\
\beta_{20}+\beta_{21}+\beta_{2(1 * F)} \\
+\beta_{2(1 * M)}+\beta_{2(F * M)}\end{array}$ & 0,33 & 5,45 \\
\hline avançado & inicial & preferencial & treinada & $\beta_{20}+\beta_{22}$ & 1,27 & 0,83 \\
\hline avançado & inicial & preferencial & controle & $\beta_{20}+\beta_{22}$ & 1,27 & 0,83 \\
\hline avançado & inicial & não & treinada & $\beta_{20}+\beta_{22}$ & 1,27 & 0,83 \\
\hline avançado & inicial & não & controle & $\beta_{20}+\beta_{22}$ & 1,27 & 0,83 \\
\hline avançado & final & preferencial & treinada & $\beta_{20}+\beta_{22}$ & 1,27 & 0,83 \\
\hline avançado & final & preferencial & controle & $\beta_{20}+\beta_{22}$ & 1,27 & 0,83 \\
\hline avançado & final & não & treinada & $\beta_{20}+\beta_{22}+\beta_{2(F * M)}$ & 0,29 & 0,37 \\
\hline avançado & final & não & controle & $\beta_{20}+\beta_{22}+\beta_{2(F * M)}$ & 0,29 & 0,37 \\
\hline
\end{tabular}


Considerando os resultados apresentados nas Tabelas 3.1 e 3.3 para os parâmetros $\theta_{j k m n}$ relacionados à dispersão, pode-se concluir que:

- fixando as outras variáveis, não há diferença entre as variabilidades da seqüência treinada e controle $\left(\beta_{2 C}=0\right)$;

- para indivíduos do grupo controle, as variabilidades são iguais $\left(\beta_{2 C}, \beta_{2 M}, \beta_{2 F}=0\right)$, exceto na avaliação final usando a mão não preferencial, em que a variabilidade é menor que as demais $\left(\beta_{2(F * M)}<0\right)$;

- para indivíduos no estágio inicial da doença, a variabilidade é menor em relação àquela de indivíduos do grupo controle $\left(\beta_{21}<0\right)$, porém variando para cada combinação de avaliação e uso de mão preferencial $\left(\beta_{2(1 * F)}, \beta_{2(1 * M)}, \beta_{2(F * M)} \neq 0\right)$;

- para indivíduos no estágio avançado da doença, a variabilidade é maior em relação àquela de indivíduos do grupo controle $\left(\beta_{22}>0\right)$, porém variando para a avaliação final usando a mão não preferencial $\left(\beta_{2(F * M)} \neq 0\right)$.

\subsection{Modelagem do total de tentativas}

Para avaliar o efeito dos fatores de interesse no total de tentativas, inicialmente foi ajustado um modelo com todos os efeitos principais e interações de primeira ordem. Após o ajuste desse modelo, testaram-se através da razão de verossimilhanças, hipóteses do tipo

$$
H_{0}: \beta_{s r}=0
$$

para verificar quais parâmetros eram significantes. Inicialmente testaram-se as interações e, em seguida, os efeitos principais.

Assim, inicialmente foi ajustado o modelo com parametrização de casela de referência, em que os vetores de parâmetros são:

$$
\boldsymbol{\beta}_{s}=\left(\beta_{s 0}, \beta_{s 1}, \beta_{s 2}, \beta_{s M}, \beta_{s(1 * M)}, \beta_{s(2 * M)}\right), \quad s=1, \ldots, 5
$$

Neste contexto 
- $\beta_{10}$ é o logaritmo do valor esperado do total de tentativas de um indivíduo do grupo controle, usando a mão preferencial, realizando a seqüência treinada na avaliação inicial;

- $\beta_{11}$ é a diferença entre os logaritmos dos valores esperados do total de tentativas de indivíduos em estágio inicial da doença e os controles, realizando a seqüência treinada na avaliação inicial;

- $\beta_{12}$ é a diferença entre os logaritmos dos valores esperados do total de tentativas de indivíduos em estágio avançado da doença e os controles, realizando a seqüência treinada na avaliação inicial;

- $\beta_{1 M}$ é a diferença entre os logaritmos dos valores esperados do total de tentativas de indivíduos usando a mão não preferencial e a preferencial, realizando a seqüência treinada na avaliação inicial.

De maneira análoga, pode-se interpretar os elementos dos vetores $\boldsymbol{\beta}_{2}, \boldsymbol{\beta}_{3}, \boldsymbol{\beta}_{4}$ (relacionados, respectivamente, aos valores esperados dos totais de tentativas na avaliação inicial realizando a seqüência controle e na avaliação final realizando as seqüências treinada e controle) e $\boldsymbol{\beta}_{5}$ (relacionados aos valores esperados para as covariâncias entre as contagens).

Com o modelo ajustado, inicialmente verificaram-se quais interações eram significantes através de um teste da hipótese

$$
\begin{aligned}
H_{0}: & \beta_{1(1 * M)}, \beta_{2(1 * M)}, \beta_{3(1 * M)}, \\
& \beta_{4(1 * M)}, \beta_{4(2 * M)}, \beta_{5(1 * M)}, \beta_{5(2 * M)}=0
\end{aligned}
$$

obtendo-se $\mathrm{p}=0,719$; os parâmetros correspondentes foram eliminados e um modelo reduzido foi ajustado. A partir desse modelo, verificou-se quais efeitos principais eram significantes através de um teste da hipótese

$$
H_{0}: \beta_{1 M}, \beta_{2 M}, \beta_{3 M}, \beta_{4 M}, \beta_{5 M}=0
$$

obtendo-se $\mathrm{p}=0,763$; também neste caso foram eliminados tais parâmetros do modelo e ajustado um novo modelo reduzido, obtendo-se então, as estimativas apresentadas na Tabela 3.4 . 
Tabela 3.4: Estimativas dos parâmetros dos vetores $\boldsymbol{\beta}_{s}(s=1, \ldots, 5)$, do modelo Poisson Tetravariado (3.1)

\begin{tabular}{|c|c|c|c|}
\hline Parâmetro & Relacionado ao & Estimativa & Erro Padrão \\
\hline \multirow[t]{2}{*}{$\boldsymbol{\beta}_{1}$} & vetor de valores esperados do total de tentativas na & & \\
\hline & avaliação inicial e seqüência treinada & & \\
\hline$\beta_{10}$ & grupo controle, mão preferencial & 2,93 & 0,05 \\
\hline$\beta_{11}$ & efeito da doença em estágio inicial & $-0,33$ & 0,09 \\
\hline$\beta_{12}$ & efeito da doença em estágio avançado & $-0,87$ & 0,13 \\
\hline$\beta_{1(2 * M)}$ & interação: estágio avançado e mão não preferencial & 0,67 & 0,17 \\
\hline \multirow[t]{2}{*}{$\boldsymbol{\beta}_{2}$} & vetor de valores esperados do total de tentativas na & & \\
\hline & avaliação inicial e seqüência controle & & \\
\hline$\beta_{20}$ & grupo controle, mão preferencial & 2,99 & 0,05 \\
\hline$\beta_{21}$ & efeito da doença em estágio inicial & $-0,26$ & 0,09 \\
\hline$\beta_{22}$ & efeito da doença em estágio avançado & $-1,03$ & 0,14 \\
\hline$\beta_{2(2 * M)}$ & interação: estágio avançado e mão não preferencial & 0,56 & 0,19 \\
\hline \multirow[t]{2}{*}{$\boldsymbol{\beta}_{3}$} & vetor de valores esperados do total de tentativas na & & \\
\hline & avaliação final e seqüência treinada & & \\
\hline$\beta_{30}$ & grupo controle, mão preferencial & 3,52 & 0,04 \\
\hline$\beta_{31}$ & efeito da doença em estágio inicial & $-0,47$ & 0,07 \\
\hline$\beta_{32}$ & efeito da doença em estágio avançado & $-0,83$ & 0,10 \\
\hline$\beta_{3(2 * M)}$ & interação: estágio avançado e mão não preferencial & 0,47 & 0,13 \\
\hline \multirow[t]{2}{*}{$\boldsymbol{\beta}_{4}$} & vetor de valores esperados do total de tentativas na & & \\
\hline & avaliação final e seqüência controle & & \\
\hline$\beta_{40}$ & grupo controle, mão preferencial & 3,31 & 0,04 \\
\hline$\beta_{41}$ & efeito da doença em estágio inicial & $-0,39$ & 0,08 \\
\hline$\beta_{42}$ & efeito da doença em estágio avançado & $-0,82$ & 0,09 \\
\hline $\boldsymbol{\beta}_{5}$ & vetor de valores esperados da covariância & & \\
\hline$\beta_{50}$ & grupo controle, mão preferencial & 2,28 & 0,14 \\
\hline$\beta_{51}$ & efeito da doença em estágio inicial & $-1,07$ & 0,48 \\
\hline$\beta_{52}$ & efeito da doença em estágio avançado & $-1,36$ & 0,38 \\
\hline
\end{tabular}


Após retirar do modelo os parâmetros nulos, testamos a igualdade dos parâmetros nas diferentes situações de contagem (avaliação inicial e final, seqüência treinada e controle), visando avaliar se o treinamento fez efeito e como esse efeito ocorre para os diferentes estágios da doença nas diferentes avaliações. Com esse objetivo, foi verificado se:

- na avaliação inicial, não há diferença entre os valores esperados dos totais de tentativas das seqüências treinada e controle;

- na avaliação final, o efeito do estágio da doença é o mesmo para as seqüências treinada e controle;

- as covariâncias entre as contagens dos doentes em estágio inicial são iguais às covariâncias entre as contagens dos doentes em estágio avançado.

Mais especificamente as hipóteses descritas acima correspondem a

$$
\begin{aligned}
H_{0}: & \boldsymbol{\beta}_{1}=\boldsymbol{\beta}_{2}, \\
\beta_{31} & =\beta_{41}, \quad \beta_{32}=\beta_{42}, \\
\beta_{51} & =\beta_{52}
\end{aligned}
$$

sob o modelo (3.1).

A validade das hipóteses acima equivale a ajustar um modelo em que

$$
\begin{aligned}
& \lambda_{j k I S}=\lambda_{j k I C}=\exp \left(\mathbf{z}_{6 i j k}^{\prime} \boldsymbol{\beta}_{6}\right) \\
& \lambda_{j k F S}=\exp \left(\mathbf{z}_{7 i j k}^{\prime} \boldsymbol{\beta}_{7}+\mathbf{z}_{8 i j k}^{\prime} \boldsymbol{\beta}_{8}\right) \\
& \lambda_{j k F C}=\exp \left(\mathbf{z}_{9 i j k}^{\prime} \boldsymbol{\beta}_{9}+\mathbf{z}_{8 i j k}^{\prime} \boldsymbol{\beta}_{8}\right) \\
& \lambda_{j k}=\exp \left(\mathbf{z}_{10, i j k}^{\prime} \boldsymbol{\beta}_{10}\right)
\end{aligned}
$$

com:

$$
\begin{aligned}
& \boldsymbol{\beta}_{6}=\left(\beta_{60}, \beta_{61}, \beta_{62}, \beta_{6(2 * M)}\right)^{\prime} \\
& \boldsymbol{\beta}_{7}=\left(\beta_{70}, \beta_{7(2 * M)}\right)^{\prime} \\
& \boldsymbol{\beta}_{8}=\left(\beta_{81}, \beta_{82}\right)^{\prime} \\
& \boldsymbol{\beta}_{9}=\left(\beta_{90}\right) \\
& \boldsymbol{\beta}_{10}=\left(\beta_{10,0}, \beta_{10, D}\right)^{\prime}
\end{aligned}
$$


que corresponde a considerar

$$
\begin{aligned}
& \boldsymbol{\beta}_{6}=\boldsymbol{\beta}_{1}=\boldsymbol{\beta}_{2} \\
& \boldsymbol{\beta}_{7}=\left(\beta_{30}, \beta_{3(2 * M)}\right)^{\prime} \\
& \boldsymbol{\beta}_{8}=\left(\beta_{31}, \beta_{32}\right)^{\prime}=\left(\beta_{41}, \beta_{42}\right)^{\prime} \\
& \boldsymbol{\beta}_{9}=\left(\beta_{40}\right) \\
& \boldsymbol{\beta}_{10}=\left(\beta_{50}, \beta_{51}\right)^{\prime}=\left(\beta_{50}, \beta_{52}\right)^{\prime}
\end{aligned}
$$

A partir do teste dessa hipótese, obteve-se $\mathrm{p}=0,500$, o que leva a não rejeitar $H_{0} . \mathrm{O}$ modelo (3.2) foi adotado como modelo final. Na Tabela 3.5 encontram-se as estimativas dos parâmetros para o modelo (3.2).

Os parâmetros do modelo (3.2) foram estimados de maneira semelhante ao modelo inicial baseado na distribuição Poisson Tetravariada (3.1), através do processo iterativo de Newton-Raphson. Os detalhes das primeiras e segundas derivadas encontram-se no Apêndice B, o código implementado no pacote S-Plus, no endereço http:/ /www.ime.usp.br/ mayra e os detalhes de como usá-lo, no Apêndice C.

A partir das estimativas da Tabela 3.5 podem-se calcular os valores esperados para os totais de tentativas $\lambda_{j k m n}$ (Tabela 3.6) e para as covariâncias $\lambda_{j k}$ (Tabela 3.7). Com base nesses valores apresentados na Tabela 3.6 e os resultados da Tabela 3.5, pode-se concluir que:

- na avaliação inicial, não há diferença entre o valor esperado das contagens das seqüências treinada e controle $\left(\boldsymbol{\beta}_{6}=\boldsymbol{\beta}_{1}=\boldsymbol{\beta}_{2}\right)$;

- na avaliação final, o efeito do estágio da doença é o mesmo para as seqüências treinada e controle $\left(\beta_{81}=\beta_{31}=\beta_{41} ; \beta_{82}=\beta_{32}=\beta_{42}\right)$;

- há efeito de treinamento, já que o número esperado de tentativas na avaliação inicial é menor que na final; e na avaliação final, o número esperado de tentativas da seqüência treinada é maior que o da seqüência controle $\left(\beta_{60}<\beta_{90}<\beta_{70}\right)$;

- tanto na avaliação inicial como na final, o total de tentativas esperado dos indivíduos doentes é menor do que dos controles; e os indivíduos em estágio avançado da doença tentam ainda menos do que os em estágio inicial $\left(\beta_{62}<\beta_{61}<0 ; \beta_{82}<\beta_{81}<0\right)$; 
Tabela 3.5: Estimativas dos parâmetros dos vetores $\boldsymbol{\beta}_{s}(s=1, \ldots, 5)$, do modelo Poisson Tetravariado final (3.2)

\begin{tabular}{llcc}
\hline Parâmetro & Relacionado ao & Estimativa & Erro Padrão \\
\hline $\boldsymbol{\beta}_{6}$ & vetor de valores esperados do total de tentativas na & & \\
& avaliação inicial & 2,96 & 0,04 \\
$\beta_{60}$ & grupo controle, mão preferencial & $-0,30$ & 0,07 \\
$\beta_{61}$ & efeito da doença em estágio inicial & $-0,94$ & 0,11 \\
$\beta_{62}$ & efeito da doença em estágio avançado & 0,62 & 0,14 \\
$\beta_{6(2 * M)}$ & interação: estágio avançado e mão não preferencial & & \\
\hline $\boldsymbol{\beta}_{7}$ & vetor de valores esperados do total de tentativas na & & \\
& avaliação final e seqüência treinada & 3,51 & 0,04 \\
$\beta_{70}$ & grupo controle, mão preferencial & 0,47 & 0,12 \\
$\beta_{7(2 * M)}$ & interação: estágio avançado e mão não preferencial & & \\
\hline $\boldsymbol{\beta}_{8}$ & vetor de valores esperados do total de tentativas na & & \multirow{2}{*}{0,06} \\
$\beta_{81}$ & avaliação final & $-0,44$ & 0,07 \\
$\beta_{82}$ & efeito da doença em estágio inicial & $-0,82$ & \\
\hline $\boldsymbol{\beta}_{9}$ & efeito da doença em estágio avançado & & \\
$\beta_{90}$ & vetor de valores esperados do total de tentativas na & & 0,15 \\
$\boldsymbol{\beta}_{10}$ & avaliação final e seqüência controle & 3,33 & 0,04 \\
\hline$\beta_{10, D}$ & vetor de valores esperados da covariância & & \\
\hline
\end{tabular}

- na avaliação inicial e para a seqüência treinada na avaliação final, o total de tentativas esperado de indivíduos em estágio avançado da doença é maior quando eles estão usando a mão não preferencial $\left(\beta_{6(2 * M)}>0 ; \beta_{7(2 * M)}>0\right)$.

Uma das suposições do modelo proposto (2.3) é que as 6 covariâncias entre contagens nos diferentes instantes de avaliação (inicial e final) e das diferentes seqüências (treinada e controle) sejam iguais. Através do vetor $\boldsymbol{\beta}_{5}$, foi permitido que os valores dessas covariâncias para cada grupo de estudo fossem diferentes. Assim, considerando os resultados do modelo Poisson Tetravariado final apresentado na Tabela 3.5 e os valores 
Tabela 3.6: Estimativas dos valores esperados do total de tentativas dos grupos de estudo nas diferentes situações de realização das seqüências

\begin{tabular}{|c|c|c|c|c|c|c|}
\hline $\begin{array}{c}\text { Estágio } \\
\text { da doença }\end{array}$ & Avaliação & Mão & Seqüência & Parâmetros & $\begin{array}{c}\text { Valor } \\
\text { esperado }\end{array}$ & $\begin{array}{l}\text { Erro } \\
\text { padrão }\end{array}$ \\
\hline controle & inicial & preferencial & treinada & $\beta_{60}$ & 19,3 & 0,8 \\
\hline controle & inicial & preferencial & controle & $\beta_{60}$ & 19,3 & 0,8 \\
\hline controle & inicial & não & treinada & $\beta_{60}$ & 19,3 & 0,8 \\
\hline controle & inicial & não & controle & $\beta_{60}$ & 19,3 & 0,8 \\
\hline controle & final & preferencial & treinada & $\beta_{70}$ & 33,4 & 1,3 \\
\hline controle & final & preferencial & controle & $\beta_{90}$ & 27,9 & 1,1 \\
\hline controle & final & não & treinada & $\beta_{70}$ & 33,4 & 1,3 \\
\hline controle & final & não & controle & $\beta_{90}$ & 27,9 & 1,1 \\
\hline inicial & inicial & preferencial & treinada & $\beta_{60}+\beta_{61}$ & 14,3 & 1,6 \\
\hline inicial & inicial & preferencial & controle & $\beta_{60}+\beta_{61}$ & 14,3 & 1,6 \\
\hline inicial & inicial & não & treinada & $\beta_{60}+\beta_{61}$ & 14,3 & 1,6 \\
\hline inicial & inicial & não & controle & $\beta_{60}+\beta_{61}$ & 14,3 & 1,6 \\
\hline inicial & final & preferencial & treinada & $\beta_{70}+\beta_{81}$ & 21,5 & 2,2 \\
\hline inicial & final & preferencial & controle & $\beta_{90}+\beta_{81}$ & 18,0 & 1,8 \\
\hline inicial & final & não & treinada & $\beta_{70}+\beta_{81}$ & 21,5 & 2,2 \\
\hline inicial & final & não & controle & $\beta_{90}+\beta_{81}$ & 18,0 & 1,8 \\
\hline avançado & inicial & preferencial & treinada & $\beta_{60}+\beta_{62}$ & 7,5 & 1,1 \\
\hline avançado & inicial & preferencial & controle & $\beta_{60}+\beta_{62}$ & 7,5 & 1,1 \\
\hline avançado & inicial & não & treinada & $\beta_{60}+\beta_{62}+\beta_{6(2 * M)}$ & 14,0 & 4,1 \\
\hline avançado & inicial & não & controle & $\beta_{60}+\beta_{62}+\beta_{6(2 * M)}$ & 14,0 & 4,1 \\
\hline avançado & final & preferencial & treinada & $\beta_{70}+\beta_{82}$ & 14,7 & 1,6 \\
\hline avançado & final & preferencial & controle & $\beta_{90}+\beta_{82}$ & 12,3 & 1,4 \\
\hline avançado & final & não & treinada & $\beta_{70}+\beta_{82}+\beta_{7(2 * M)}$ & 23,6 & 5,5 \\
\hline avançado & final & não & controle & $\beta_{90}+\beta_{82}$ & 12,3 & 1,4 \\
\hline
\end{tabular}


Tabela 3.7: Estimativas dos valores esperados das covariâncias entre as contagens de totais de tentativas das duas seqüências nas duas avaliações, para os 6 grupos de estudo

\begin{tabular}{cclcc}
\hline $\begin{array}{c}\text { Estágio } \\
\text { da doença }\end{array}$ & Mão & Parâmetros & $\begin{array}{c}\text { Valor } \\
\text { esperado }\end{array}$ & $\begin{array}{c}\text { Erro } \\
\text { padrão }\end{array}$ \\
\hline controle & preferencial & $\beta_{10,0}$ & 9,7 & 1,5 \\
controle & não & $\beta_{10,0}$ & 9,7 & 1,5 \\
\hline inicial & preferencial & $\beta_{10,0}+\beta_{10, D}$ & 2,6 & 1,3 \\
inicial & não & $\beta_{10,0}+\beta_{10, D}$ & 2,6 & 1,3 \\
\hline avançado & preferencial & $\beta_{10,0}+\beta_{10, D}$ & 2,6 & 1,3 \\
avançado & não & $\beta_{10,0}+\beta_{10, D}$ & 2,6 & 1,3 \\
\hline
\end{tabular}

esperados para as covariâncias (Tabela 3.7), pode-se concluir que:

- a covariância esperada entre contagens de totais de tentativas das duas seqüências nas duas avaliações é a mesma para indivíduos usando ou não a mão preferencial $\left(\beta_{5 M}=0\right)$;

- a covariância esperada entre contagens de totais de tentativas das duas seqüências nas duas avaliações de indivíduos nos dois estágios da doença é a mesma $\left(\beta_{10, D}=\right.$ $\left.\beta_{51}=\beta_{52}\right)$; e essa covariância é menor do que aquela para indivíduos do grupo controle $\left(\beta_{10, D}<0\right)$.

\subsection{Resultados finais e sugestões}

A partir das estimativas obtidas nas seções 3.1 e 3.2, foi construída a Tabela 3.8 com os valores esperados para as contagens de acertos e totais. Pode-se notar que houve efeito de treinamento, aumentando tanto o número de acertos quanto o total de tentativas. O único caso em que houve diminuição do número de acertos e de total de tentativas foi para os doentes em estágio avançado realizando a seqüência controle com a mão não preferencial. Nota-se também que, o total de tentativas com a seqüência treinada na avaliação final é maior que o obtido com a seqüência controle.

Para todas as situações, observa-se que o total de tentativas é maior para os indivíduos do grupo controle; entre os doentes, aqueles em estágio inicial realizam mais 
Tabela 3.8: Estimativas dos valores esperados do número de acertos e total de tentativas dos grupos de estudo nas diferentes situações de realização das seqüências

\begin{tabular}{|c|c|c|c|c|c|}
\hline Estágio & Avaliação & Mão & Seqüência & Acertos (EP) & Totais (EP) \\
\hline controle & inicial & preferencial & treinada & $16,4(1,1)$ & $19,3(0,8)$ \\
\hline controle & inicial & preferencial & controle & $16,4(1,1)$ & $19,3(0,8)$ \\
\hline controle & inicial & não & treinada & $16,4(1,1)$ & $19,3(0,8)$ \\
\hline controle & inicial & não & controle & $16,4(1,1)$ & $19,3(0,8)$ \\
\hline controle & final & preferencial & treinada & $32,1(1,9)$ & $33,4(1,3)$ \\
\hline controle & final & preferencial & controle & $22,6(4,2)$ & $27,9(1,1)$ \\
\hline controle & final & não & treinada & $32,1(1,9)$ & $33,4(1,3)$ \\
\hline controle & final & não & controle & $22,6(4,2)$ & $27,9(1,1)$ \\
\hline inicial & inicial & preferencial & treinada & $12,2(1,6)$ & $14,3(1,6)$ \\
\hline inicial & inicial & preferencial & controle & $12,2(1,6)$ & $14,3(1,6)$ \\
\hline inicial & inicial & não & treinada & $12,2(1,6)$ & $14,3(1,6)$ \\
\hline inicial & inicial & não & controle & $12,2(1,6)$ & $14,3(1,6)$ \\
\hline inicial & final & preferencial & treinada & $20,6(2,5)$ & $21,5(2,2)$ \\
\hline inicial & final & preferencial & controle & $14,6(3,6)$ & $18,0(1,8)$ \\
\hline inicial & final & não & treinada & $20,6(2,5)$ & $21,5(2,2)$ \\
\hline inicial & final & não & controle & $14,6(3,6)$ & $18,0(1,8)$ \\
\hline avançado & inicial & preferencial & treinada & $4,7(1,4)$ & $7,5(1,1)$ \\
\hline avançado & inicial & preferencial & controle & $4,7(1,4)$ & $7,5(1,1)$ \\
\hline avançado & inicial & não & treinada & $8,7(3,8)$ & $14,0(4,1)$ \\
\hline avançado & inicial & não & controle & $8,7(3,8)$ & $14,0(4,1)$ \\
\hline avançado & final & preferencial & treinada & $12,9(2,4)$ & $14,7(1,6)$ \\
\hline avançado & final & preferencial & controle & $6,6(3,5)$ & $12,3(1,4)$ \\
\hline avançado & final & não & treinada & $20,8(6,5)$ & $23,6(5,5)$ \\
\hline avançado & final & não & controle & $6,6(3,5)$ & $12,3(1,4)$ \\
\hline
\end{tabular}


tentativas que os em estágio avançado, com exceção da seqüência treinada na avaliação final, usando a mão não preferencial.

A distribuição Poisson Multivariada apresentada, por exemplo, em Kawamura (1979) permite que as covariâncias entre duas contagens de totais de tentativas sejam todas diferentes entre si, ao contrário do modelo (2.3), que assume covariâncias iguais entre as 4 contagens. Os dados sugerem que não há problemas em fazer tal suposição, já que, em um mesmo grupo de estudo, as covariâncias amostrais são muito próximas (a maior é em torno de 4 vezes a menor). A exceção ocorre para as covariâncias entre as contagens dos doentes em estágio avançado, usando a mão preferencial, caso em que a maior covariância é 12 vezes a menor. Em futuros trabalhos, poderiam ser adotados modelos que permitissem que as covariâncias fossem diferentes, porém acarretando em um grande aumento no número de parâmetros.

Uma outra possibilidade seria propor um modelo como o sugerido em Zhu et al. (2003), no qual é incorporado um parâmetro para tentar relacionar os valores esperados da probabilidade de acerto e do total de tentativas.

Além disso, para considerar a sobredispersão sugerida pelos dados (Tabela 1.1), poderia ser proposto um modelo de efeitos aleatórios também para a contagem de totais. Poderiam também ser usadas técnicas Bayesianas, ou ainda outros algoritmos de maximização, como o EM (expectation maximization) utilizado em Zhu et al. (2003). 


\section{Apêndice A}

\section{Correlações amostrais}

Tabela A.1: Correlações entre as contagens para indivíduos do grupo controle usando a mão preferencial

\begin{tabular}{|c|c|c|c|c|c|c|c|c|c|c|}
\hline & & & \multicolumn{4}{|c|}{ Inicial } & \multicolumn{4}{|c|}{ Final } \\
\hline & & & \multicolumn{2}{|c|}{ Treinada } & \multicolumn{2}{|c|}{ Controle } & \multicolumn{2}{|c|}{ Treinada } & \multicolumn{2}{|c|}{ Controle } \\
\hline & & & Acerto & Total & Acerto & Total & Acerto & Total & Acerto & Total \\
\hline \multirow[t]{4}{*}{ Inicial } & Treinada & Acertos & 1 & & & & & & & \\
\hline & & Totais & 0,99 & 1 & & & & & & \\
\hline & Controle & Acertos & 0,85 & 0,84 & 1 & & & & & \\
\hline & & Totais & 0,78 & 0,80 & 0,96 & 1 & & & & \\
\hline \multirow[t]{4}{*}{ Final } & Treinada & Acertos & 0,76 & 0,76 & 0,61 & 0,61 & 1 & & & \\
\hline & & Totais & 0,74 & 0,74 & 0,61 & 0,63 & 0,99 & 1 & & \\
\hline & Controle & Acertos & 0,53 & 0,49 & 0,59 & 0,63 & 0,60 & 0,61 & 1 & \\
\hline & & Totais & 0,81 & 0,82 & 0,70 & 0,69 & 0,93 & 0,92 & 0,50 & 1 \\
\hline
\end{tabular}


Tabela A.2: Correlações entre as contagens para indivíduos do grupo controle usando a mão não preferencial

\begin{tabular}{|c|c|c|c|c|c|c|c|c|c|c|}
\hline & & & \multicolumn{4}{|c|}{ Inicial } & \multicolumn{4}{|c|}{ Final } \\
\hline & & & \multicolumn{2}{|c|}{ Treinada } & \multicolumn{2}{|c|}{ Controle } & \multicolumn{2}{|c|}{ Treinada } & \multicolumn{2}{|c|}{ Controle } \\
\hline & & & Acerto & Total & Acerto & Total & Acerto & Total & Acerto & Total \\
\hline \multirow[t]{4}{*}{ Inicial } & Treinada & Acertos & 1 & & & & & & & \\
\hline & & Totais & 0,73 & 1 & & & & & & \\
\hline & Controle & Acertos & 0,76 & 0,53 & 1 & & & & & \\
\hline & & Totais & 0,57 & 0,86 & 0,71 & 1 & & & & \\
\hline \multirow[t]{4}{*}{ Final } & Treinada & Acertos & 0,73 & 0,29 & 0,88 & 0,38 & 1 & & & \\
\hline & & Totais & 0,67 & 0,28 & 0,85 & 0,37 & 0,97 & 1 & & \\
\hline & Controle & Acertos & 0,63 & 0,43 & 0,64 & 0,42 & 0,71 & 0,66 & 1 & \\
\hline & & Totais & 0,83 & 0,54 & 0,82 & 0,51 & 0,82 & 0,76 & 0,89 & 1 \\
\hline
\end{tabular}

Tabela A.3: Correlações entre as contagens dos doentes em estágio inicial usando a mão preferencial

\begin{tabular}{|c|c|c|c|c|c|c|c|c|c|c|}
\hline & & & \multicolumn{4}{|c|}{ Inicial } & \multicolumn{4}{|c|}{ Final } \\
\hline & & & \multicolumn{2}{|c|}{ Treinada } & \multicolumn{2}{|c|}{ Controle } & \multicolumn{2}{|c|}{ Treinada } & \multicolumn{2}{|c|}{ Controle } \\
\hline & & & Acerto & Total & Acerto & Total & Acerto & Total & Acerto & Total \\
\hline \multirow[t]{4}{*}{ Inicial } & Treinada & Acertos & 1 & & & & & & & \\
\hline & & Totais & 0,95 & 1 & & & & & & \\
\hline & Controle & Acertos & 0,00 & 0,09 & 1 & & & & & \\
\hline & & Totais & 0,01 & 0,13 & 0,97 & 1 & & & & \\
\hline \multirow[t]{4}{*}{ Final } & Treinada & Acertos & 0,49 & 0,57 & $-0,26$ & $-0,26$ & 1 & & & \\
\hline & & Totais & 0,44 & 0,48 & $-0,43$ & $-0,52$ & 0,84 & 1 & & \\
\hline & Controle & Acertos & 0,46 & 0,33 & 0,58 & 0,51 & 0,09 & $-0,14$ & 1 & \\
\hline & & Totais & 0,47 & 0,34 & 0,46 & 0,30 & 0,27 & 0,23 & 0,88 & 1 \\
\hline
\end{tabular}


Tabela A.4: Correlações entre as contagens dos doentes em estágio inicial usando a mão não preferencial

\begin{tabular}{|c|c|c|c|c|c|c|c|c|c|c|}
\hline & & & \multicolumn{4}{|c|}{ Inicial } & \multicolumn{4}{|c|}{ Final } \\
\hline & & & \multicolumn{2}{|c|}{ Treinada } & \multicolumn{2}{|c|}{ Controle } & \multicolumn{2}{|c|}{ Treinada } & \multicolumn{2}{|c|}{ Controle } \\
\hline & & & Acerto & Total & Acerto & Total & Acerto & Total & Acerto & Total \\
\hline \multirow[t]{4}{*}{ Inicial } & Treinada & Acertos & 1 & & & & & & & \\
\hline & & Totais & 0,67 & 1 & & & & & & \\
\hline & Controle & Acertos & 0,79 & 0,37 & 1 & & & & & \\
\hline & & Totais & 0,69 & 0,59 & 0,85 & 1 & & & & \\
\hline \multirow[t]{4}{*}{ Final } & Treinada & Acertos & 0,71 & 0,30 & 0,73 & 0,62 & 1 & & & \\
\hline & & Totais & 0,70 & 0,29 & 0,69 & 0,61 & 0,99 & 1 & & \\
\hline & Controle & Acertos & 0,59 & 0,26 & 0,61 & 0,57 & 0,83 & 0,85 & 1 & \\
\hline & & Totais & 0,54 & 0,28 & 0,48 & 0,57 & 0,79 & 0,84 & 0,94 & 1 \\
\hline
\end{tabular}

Tabela A.5: Correlações entre as contagens dos doentes em estágio avançado usando a mão preferencial

\begin{tabular}{|c|c|c|c|c|c|c|c|c|c|c|}
\hline & & & \multicolumn{4}{|c|}{ Inicial } & \multicolumn{4}{|c|}{ Final } \\
\hline & & & \multicolumn{2}{|c|}{ Treinada } & \multicolumn{2}{|c|}{ Controle } & \multicolumn{2}{|c|}{ Treinada } & \multicolumn{2}{|c|}{ Controle } \\
\hline & & & Acerto & Total & Acerto & Total & Acerto & Total & Acerto & Total \\
\hline \multirow[t]{4}{*}{ Inicial } & Treinada & Acertos & 1 & & & & & & & \\
\hline & & Totais & 0,55 & 1 & & & & & & \\
\hline & Controle & Acertos & 0,38 & 0,51 & 1 & & & & & \\
\hline & & Totais & $-0,03$ & 0,45 & 0,82 & 1 & & & & \\
\hline \multirow[t]{4}{*}{ Final } & Treinada & Acertos & 0,09 & 0,52 & 0,83 & 0,73 & 1 & & & \\
\hline & & Totais & $-0,08$ & 0,45 & 0,79 & 0,78 & 0,97 & 1 & & \\
\hline & Controle & Acertos & $-0,11$ & 0,38 & 0,68 & 0,55 & 0,89 & 0,89 & 1 & \\
\hline & & Totais & $-0,05$ & 0,49 & 0,76 & 0,70 & 0,98 & 0,97 & 0,95 & 1 \\
\hline
\end{tabular}


Tabela A.6: Correlações entre as contagens dos doentes em estágio avançado usando a mão não preferencial

\begin{tabular}{|c|c|c|c|c|c|c|c|c|c|c|}
\hline & & & \multicolumn{4}{|c|}{ Inicial } & \multicolumn{4}{|c|}{ Final } \\
\hline & & & \multicolumn{2}{|c|}{ Treinada } & \multicolumn{2}{|c|}{ Controle } & \multicolumn{2}{|c|}{ Treinada } & \multicolumn{2}{|c|}{ Controle } \\
\hline & & & Acerto & Total & Acerto & Total & Acerto & Total & Acerto & Total \\
\hline \multirow[t]{4}{*}{ Inicial } & Treinada & Acertos & 1 & & & & & & & \\
\hline & & Totais & 0,99 & 1 & & & & & & \\
\hline & Controle & Acertos & 0,95 & 0,89 & 1 & & & & & \\
\hline & & Totais & 0,94 & 0,88 & 0,96 & 1 & & & & \\
\hline \multirow[t]{4}{*}{ Final } & Treinada & Acertos & 0,74 & 0,78 & 0,68 & 0,51 & 1 & & & \\
\hline & & Totais & 0,59 & 0,66 & 0,51 & 0,31 & 0,98 & 1 & & \\
\hline & Controle & Acertos & 0,05 & $-0,08$ & 0,33 & 0,12 & 0,22 & 0,16 & 1 & \\
\hline & & Totais & $-0,62$ & $-0,58$ & $-0,56$ & $-0,78$ & 0,05 & 0,22 & 0,37 & 1 \\
\hline
\end{tabular}




\section{Apêndice B}

\section{Expressões das derivadas do modelo Beta-Binomial/Poisson}

\section{B.1 Modelo Beta-Binomial (2.9)}

B.1.1 Primeiras derivadas

$$
\begin{aligned}
& \text { (i) } \frac{\partial L}{\partial \beta_{1 r_{1}}}=\sum_{j=0}^{2} \sum_{i=1}^{m_{j}} \sum_{m=I}^{F} \sum_{n=S}^{C} a_{i j k m n} \mu_{j k m n}\left(1-\mu_{j k m n}\right) z_{1 i j k m n r_{1}} \\
& \text { (ii) } \frac{\partial L}{\partial \beta_{2 r_{2}}}=\sum_{j=0}^{2} \sum_{i=1}^{m_{j}} \sum_{m=I}^{F} \sum_{n=S}^{C} b_{i j k m n} \theta_{j k m n} z_{2 i j k m n r_{2}}
\end{aligned}
$$

$r_{s}=1, \ldots, R_{s} ; \mathrm{s}=1,2$.

B.1.2 Segundas derivadas

$$
\begin{aligned}
& \text { (i) } \frac{\partial^{2} L}{\partial \beta_{1 r_{1}} \partial \beta_{1 r_{1}^{\prime}}}=\sum_{j=0}^{2} \sum_{i=1}^{m_{j}} \sum_{m=I}^{F} \sum_{n=S}^{C} c_{i j k m n} \mu_{j k m n}\left(1-\mu_{j k m n}\right) z_{1 i j k m n r_{1}} z_{1 i j k m n r_{1}^{\prime}} \\
& \text { (ii) } \frac{\partial^{2} L}{\partial \beta_{2 r_{2}} \partial \beta_{2 r_{2}^{\prime}}}=\sum_{j=0}^{2} \sum_{i=1}^{m_{j}} \sum_{m=I}^{F} \sum_{n=S}^{C} d_{i j k m n} \theta_{j k m n} z_{2 i j k m n r_{2}} z_{2 i j k m n r_{2}^{\prime}}
\end{aligned}
$$


(iii) $\frac{\partial^{2} L}{\partial \beta_{1 r_{1}} \partial \beta_{2 r_{2}}}=\sum_{j=0}^{2} \sum_{i=1}^{m_{j}} \sum_{m=I}^{F} \sum_{n=S}^{C} e_{i j k m n} \mu_{j k m n}\left(1-\mu_{j k m n}\right) \theta_{j k m n} z_{1 i j k m n r_{1}} z_{2 i j k m n r_{2}}$

$r_{s}, r_{s}^{\prime}=1, \ldots, R_{s} ; \mathrm{s}=1,2$.

\section{B.2 Modelo Poisson Tetravariado completo (3.1)}

\section{B.2.1 Primeiras derivadas}
(i) $\frac{\partial L}{\partial \beta_{1 r_{1}}}=\sum_{j=0}^{2} \sum_{i=1}^{m_{j}} a_{i j k} \lambda_{j k I S} z_{1 i j k r_{1}}$
(ii) $\frac{\partial L}{\partial \beta_{2 r_{2}}}=\sum_{j=0}^{2} \sum_{i=1}^{m_{j}} b_{i j k} \lambda_{j k I C} z_{2 i j k r_{2}}$
(iii) $\frac{\partial L}{\partial \beta_{3 r_{3}}}=\sum_{j=0}^{2} \sum_{i=1}^{m_{j}} c_{i j k} \lambda_{j k F S} z_{3 i j k r_{3}}$
(iv) $\frac{\partial L}{\partial \beta_{4 r_{4}}}=\sum_{j=0}^{2} \sum_{i=1}^{m_{j}} d_{i j k} \lambda_{j k F C} z_{4 i j k r_{4}}$
(v) $\frac{\partial L}{\partial \beta_{5 r_{5}}}=\sum_{j=0}^{2} \sum_{i=1}^{m_{j}} e_{i j k} \lambda_{j k} z_{5 i j k r_{5}}$

$r_{s},=1, \ldots, R_{s} ; \mathrm{s}=1, \ldots, 5$.

\section{B.2.2 Segundas derivadas}

$$
\begin{aligned}
& \text { (i) } \frac{\partial^{2} L}{\partial \beta_{1 r_{1}} \partial \beta_{1 r_{1}^{\prime}}}=\sum_{j=0}^{2} \sum_{i=1}^{m_{j}} f_{i j k} \lambda_{j k I S} z_{1 i j k r_{1}} z_{1 i j k r_{1}^{\prime}} \\
& \text { (ii) } \frac{\partial^{2} L}{\partial \beta_{1 r_{1}} \partial \beta_{2 r_{2}}}=\sum_{j=0}^{2} \sum_{i=1}^{m_{j}} g_{i j k} \lambda_{j k I S} \lambda_{j k I C} z_{1 i j k r_{1}} z_{2 i j k r_{2}}
\end{aligned}
$$


(iii) $\frac{\partial^{2} L}{\partial \beta_{1 r_{1}} \partial \beta_{3 r_{3}}}=\sum_{j=0}^{2} \sum_{i=1}^{m_{j}} h_{i j k} \lambda_{j k I S} \lambda_{j k F S} z_{1 i j k r_{1}} z_{3 i j k r_{3}}$

(iv) $\frac{\partial^{2} L}{\partial \beta_{1 r_{1}} \partial \beta_{4 r_{4}}}=\sum_{j=0}^{2} \sum_{i=1}^{m_{j}} m_{i j k} \lambda_{j k I S} \lambda_{j k F C} z_{1 i j k r_{1}} z_{4 i j k r_{4}}$

(v) $\frac{\partial^{2} L}{\partial \beta_{1 r_{1}} \partial \beta_{5 r_{5}}}=\sum_{j=0}^{2} \sum_{i=1}^{m_{j}} n_{i j k} \lambda_{j k I S} \lambda_{j k} z_{1 i j k r_{1}} z_{5 i j k r_{5}}$

(vi) $\frac{\partial^{2} L}{\partial \beta_{2 r_{2}} \partial \beta_{2 r_{2}^{\prime}}}=\sum_{j=0}^{2} \sum_{i=1}^{m_{j}} p_{i j k} \lambda_{j k I C} z_{2 i j k r_{2}} z_{2 i j k r_{2}^{\prime}}$

(vii) $\frac{\partial^{2} L}{\partial \beta_{2 r_{2}} \partial \beta_{3 r_{3}}}=\sum_{j=0}^{2} \sum_{i=1}^{m_{j}} q_{i j k} \lambda_{j k I C} \lambda_{j k F S} z_{2 i j k r_{2}} z_{3 i j k r_{3}}$

(viii) $\frac{\partial^{2} L}{\partial \beta_{2 r_{2}} \partial \beta_{4 r_{4}}}=\sum_{j=0}^{2} \sum_{i=1}^{m_{j}} r_{i j k} \lambda_{j k I C} \lambda_{j k F C} z_{2 i j k r_{2}} z_{4 i j k r_{4}}$

(ix) $\frac{\partial^{2} L}{\partial \beta_{2 r_{2}} \partial \beta_{5 r_{5}}}=\sum_{j=0}^{2} \sum_{i=1}^{m_{j}} s_{i j k} \lambda_{j k I C} \lambda_{j k} z_{2 i j k r_{2}} z_{5 i j k r_{5}}$

(x) $\frac{\partial^{2} L}{\partial \beta_{3 r_{3}} \partial \beta_{3 r_{3}^{\prime}}}=\sum_{j=0}^{2} \sum_{i=1}^{m_{j}} t_{i j k} \lambda_{j k F S} z_{3 i j k r_{3}} z_{3 i j k r_{3}^{\prime}}$

(xi) $\frac{\partial^{2} L}{\partial \beta_{3 r_{3}} \partial \beta_{4 r_{4}}}=\sum_{j=0}^{2} \sum_{i=1}^{m_{j}} u_{i j k} \lambda_{j k F S} \lambda_{j k F C} z_{3 i j k r_{3}} z_{4 i j k r_{4}}$

(xii) $\frac{\partial^{2} L}{\partial \beta_{3 r_{3}} \partial \beta_{5 r_{5}}}=\sum_{j=0}^{2} \sum_{i=1}^{m_{j}} v_{i j k} \lambda_{j k F S} \lambda_{j k} z_{3 i j k r_{3}} z_{5 i j k r_{5}}$

(xiii) $\frac{\partial^{2} L}{\partial \beta_{4 r_{4}} \partial \beta_{4 r_{4}^{\prime}}}=\sum_{j=0}^{2} \sum_{i=1}^{m_{j}} w_{i j k} \lambda_{j k F C} z_{4 i j k r_{4}} z_{4 i j k r_{4}^{\prime}}$

(xiv) $\frac{\partial^{2} L}{\partial \beta_{4 r_{4}} \partial \beta_{5 r_{5}}}=\sum_{j=0}^{2} \sum_{i=1}^{m_{j}} x_{i j k} \lambda_{j k F C} \lambda_{j k} z_{4 i j k r_{4}} z_{5 i j k r_{5}}$

(xv) $\frac{\partial^{2} L}{\partial \beta_{5 r_{5}} \partial \beta_{5 r_{5}^{\prime}}}=\sum_{j=0}^{2} \sum_{i=1}^{m_{j}} y_{i j k} \lambda_{j k} z_{5 i j k r_{5}} z_{5 i j k r_{5}^{\prime}}$

$r_{s}, r_{s}^{\prime}=1, \ldots, R_{s} ; \mathrm{s}=1, \ldots, 5$. 


\section{B.3 Modelo Poisson Tetravariado final (3.2)}

\section{B.3.1 Primeiras derivadas}

$$
\begin{aligned}
\text { (i) } \quad \frac{\partial L}{\partial \beta_{6 r_{6}}} & =\sum_{j=0}^{2} \sum_{i=1}^{m_{j}} a_{i j k} \lambda_{j k I S} z_{6 i j k r_{6}} \\
\operatorname{com} a_{i j k} & =P_{1000}+P_{0100}-2 \\
\text { (ii) } \quad \frac{\partial L}{\partial \beta_{7 r_{7}}} & =\sum_{j=0}^{2} \sum_{i=1}^{m_{j}} b_{i j k} \lambda_{j k F S} z_{7 i j k r_{7}} \\
\operatorname{com~} b_{i j k} & =P_{0010}-1 \\
\text { (iii) } \frac{\partial L}{\partial \beta_{8 r_{8}}} & =\sum_{j=0}^{2} \sum_{i=1}^{m_{j}}\left[b_{i j k} \lambda_{j k F S}+c_{i j k} \lambda_{j k F C}\right] z_{8 i j k r_{8}} \\
\operatorname{com} c_{i j k} & =P_{0001}-1 \\
\text { (iv) } \frac{\partial L}{\partial \beta_{9 r_{9}}} & =\sum_{j=0}^{2} \sum_{i=1}^{m_{j}} c_{i j k} \lambda_{j k F C} z_{9 i j k r_{9}} \\
\text { (v) } \frac{\partial L}{\partial \beta_{10 r_{10}}} & =\sum_{j=0}^{2} \sum_{i=1}^{m_{j}} d_{i j k} \lambda_{j k} z_{10, i j k r_{10}} \\
\operatorname{com~} d_{i j k} & =3+P_{1111}-P_{1000}-P_{0100}-P_{0010}-P_{0001}
\end{aligned}
$$

$r_{s}=1, \ldots, R_{s} ; \mathrm{s}=6, \ldots, 10 ;$ que em notação matricial podem ser expressas como:

$$
\begin{gathered}
\frac{\partial L}{\partial \boldsymbol{\beta}_{6}}=\boldsymbol{Z}_{6}^{\prime} \boldsymbol{L}_{I} \mathbf{A}, \frac{\partial L}{\partial \boldsymbol{\beta}_{7}}=\boldsymbol{Z}_{7}^{\prime} \boldsymbol{L}_{F S} \mathbf{B}, \frac{\partial L}{\partial \boldsymbol{\beta}_{8}}=\boldsymbol{Z}_{8}^{\prime}\left[\boldsymbol{L}_{F S} \mathbf{B}+\boldsymbol{L}_{F C} \mathbf{C}\right], \\
\frac{\partial L}{\partial \boldsymbol{\beta}_{9}}=\boldsymbol{Z}_{9}^{\prime} \boldsymbol{L}_{F C} \mathbf{C} \text { e } \frac{\partial L}{\partial \boldsymbol{\beta}_{10}}=\boldsymbol{Z}_{10}^{\prime} \mathbf{L D}
\end{gathered}
$$

com:

$$
\begin{aligned}
& \mathbf{A}=\left(a_{10 k}, \ldots, a_{i j k}, \ldots, a_{12,2 k}\right)^{\prime}, \mathbf{B}=\left(b_{10 k}, \ldots, b_{i j k}, \ldots, b_{12,2 k}\right)^{\prime}, \\
& \mathbf{C}=\left(c_{10 k}, \ldots, c_{i j k}, \ldots, c_{12,2 k}\right)^{\prime}, \mathbf{D}=\left(d_{10 k}, \ldots, d_{i j k}, \ldots, d_{12,2 k}\right)^{\prime}, \\
& \mathbf{Z}_{s}=\left(\mathbf{z}_{s 10 k}^{\prime}, \ldots, \mathbf{z}_{s i j k}^{\prime}, \ldots, \mathbf{z}_{s, 12,2 k}^{\prime}\right), s=6, \ldots, 10, \\
& \boldsymbol{L}_{I}=\operatorname{diag}\left(\lambda_{j k I S}\right)=\operatorname{diag}\left(\lambda_{j k I C}\right), \boldsymbol{L}_{F S}=\operatorname{diag}\left(\lambda_{j k F S}\right), \boldsymbol{L}_{F C}=\operatorname{diag}\left(\lambda_{j k F C}\right) \\
& \text { e } \mathbf{L}=\operatorname{diag}\left(\lambda_{\mathrm{jk}}\right) .
\end{aligned}
$$




\section{B.3.2 Segundas derivadas}

(i) $\frac{\partial^{2} L}{\partial \beta_{6 r_{6}} \partial \beta_{6 r_{6}^{\prime}}}=\sum_{j=0}^{2} \sum_{i=1}^{m_{j}}\left[a_{i j k}+e_{i j k} \lambda_{j k I S}\right] \lambda_{j k I S} z_{1 i j k r_{1}} z_{1 i j k r_{1}^{\prime}}$

$\operatorname{com} e_{i j k}=P_{2000}+P_{0200}+2 P_{1100}-2 P_{1000} P_{0100}-P_{1000}^{2}-P_{0100}^{2}$

(ii) $\frac{\partial^{2} L}{\partial \beta_{6 r_{6}} \partial \beta_{7 r_{7}}}=\sum_{j=0}^{2} \sum_{i=1}^{m_{j}} f_{i j k} \lambda_{j k I S} \lambda_{j k F S} z_{6 i j k r_{6}} z_{7 i j k r_{7}}$

$\operatorname{com} f_{i j k}=P_{1010}+P_{0110}-P_{1000} P_{0010}-P_{0100} P_{0010}$

(iii) $\frac{\partial^{2} L}{\partial \beta_{6 r_{6}} \partial \beta_{8 r_{8}}}=\sum_{j=0}^{2} \sum_{i=1}^{m_{j}}\left[f_{i j k} \lambda_{j k F S}+g_{i j k} \lambda_{j k F C}\right] \lambda_{j k I S} z_{6 i j k r_{6}} z_{8 i j k r_{8}}$

com $g_{i j k}=P_{1001}+P_{0101}-P_{1000} P_{0001}-P_{0100} P_{0001}$

(iv) $\frac{\partial^{2} L}{\partial \beta_{6 r_{6}} \partial \beta_{9 r_{9}}}=\sum_{j=0}^{2} \sum_{i=1}^{m_{j}} g_{i j k} \lambda_{j k I S} \lambda_{j k F C} z_{6 i j k r_{6}} z_{9 i j k r_{9}}$

(v) $\frac{\partial^{2} L}{\partial \beta_{6 r_{6}} \partial \beta_{10 r_{10}}}=\sum_{j=0}^{2} \sum_{i=1}^{m_{j}} h_{i j k} \lambda_{j k I S} \lambda_{j k} z_{6 i j k r_{6}} z_{10 i j k r_{10}}$

$\operatorname{com} h_{i j k}=P_{2111}+P_{1211}-P_{2000}-P_{0200}-2 P_{1100}-P_{1010}-P_{1001}$

$-P_{0110}-P_{0101}+\left(P_{1000}+P_{0100}\right)\left(P_{1000}+P_{0100}+P_{0010}+P_{0001}-P_{1111}\right)$

$(v i)$

$\frac{\partial^{2} L}{\partial \beta_{7 r_{7}} \partial \beta_{7 r_{7}^{\prime}}}=\sum_{j=0}^{2} \sum_{i=1}^{m_{j}}\left[b_{i j k}+m_{i j k} \lambda_{j k F S}\right] \lambda_{j k F S} z_{7 i j k r_{7}} z_{7 i j k r_{7}^{\prime}}$

com $m_{i j k}=P_{0020}-P_{0010}^{2}$

(vii) $\frac{\partial^{2} L}{\partial \beta_{7 r_{7}} \partial \beta_{8 r_{8}}}=\sum_{j=0}^{2} \sum_{i=1}^{m_{j}}\left[b_{i j k}+m_{i j k} \lambda_{j k F S}+n_{i j k} \lambda_{j k F C}\right] \lambda_{j k F S} z_{7 i j k r_{7}} z_{8 i j k r_{8}}$

com $n_{i j k}=P_{0011}-P_{0010} P_{0001}$

(viii) $\frac{\partial^{2} L}{\partial \beta_{7 r_{7}} \partial \beta_{9 r_{9}}}=\sum_{j=0}^{2} \sum_{i=1}^{m_{j}} n_{i j k} \lambda_{j k F S} \lambda_{j k F C} z_{7 i j k r_{7}} z_{9 i j k r_{9}}$

(ix) $\frac{\partial^{2} L}{\partial \beta_{7 r_{7}} \partial \beta_{10 r_{10}}}=\sum_{j=0}^{2} \sum_{i=1}^{m_{j}} q_{i j k} \lambda_{j k F S} \lambda_{j k} z_{7 i j k r_{7}} z_{10 i j k r_{10}}$

$\operatorname{com} q_{i j k}=P_{1121}-P_{1010}-P_{0110}-P_{0020}-P_{0011}$

$+P_{0010}\left(P_{1000}+P_{0100}+P_{0010}+P_{0001}-P_{1111}\right)$ 
(x) $\frac{\partial^{2} L}{\partial \beta_{8 r_{8}} \partial \beta_{8 r_{8}^{\prime}}}=\sum_{j=0}^{2} \sum_{i=1}^{m_{j}} z_{8 i j k r_{8}} z_{8 i j k r_{8}^{\prime}}$

$\left[\lambda_{j k F S}\left(m_{i j k} \lambda_{j k F S}+b_{i j k}+s_{i j k} \lambda_{j k F C}\right)+\lambda_{j k F C}\left(n_{i j k} \lambda_{j k F S}+c_{i j k}+r_{i j k} \lambda_{j k F C}\right)\right]$

com $r_{i j k}=P_{0002}-P_{0001}^{2}$ e $s_{i j k}=P_{0011}-P_{0001} P_{0010}$

$\frac{\partial^{2} L}{\partial \beta_{8 r_{8}} \partial \beta_{9 r_{9}}}=\sum_{j=0}^{2} \sum_{i=1}^{m_{j}}\left[n_{i j k} \lambda_{j k F S}+c_{i j k}+r_{i j k} \lambda_{j k F C}\right] \lambda_{j k F C} z_{8 i j k r_{8}} z_{9 i j k r_{9}}$

(xii) $\frac{\partial^{2} L}{\partial \beta_{8 r_{8}} \partial \beta_{10 r_{10}}}=\sum_{j=0}^{2} \sum_{i=1}^{m_{j}}\left[q_{i j k} \lambda_{j k F S}+t_{i j k} \lambda_{j k F C}\right] \lambda_{j k} z_{8 i j k r_{8}} z_{10 i j k r_{10}}$

com $t_{i j k}=P_{1112}-P_{1001}-P_{0101}-P_{0011}-P_{0002}$

$+P_{0001}\left(P_{1000}+P_{0100}+P_{0010}+P_{0001}-P_{1111}\right)$

(xiii) $\frac{\partial^{2} L}{\partial \beta_{9 r_{9}} \partial \beta_{9 r_{9}^{\prime}}}=\sum_{j=0}^{2} \sum_{i=1}^{m_{j}}\left[c_{i j k}+u_{i j k} \lambda_{j k F C}\right] \lambda_{j k F C} z_{9 i j k r_{9}} z_{9 i j k r_{9}^{\prime}}$

com $u_{i j k}=P_{0002}-P_{0001}^{2}$

(xiv) $\frac{\partial^{2} L}{\partial \beta_{9 r_{9}} \partial \beta_{10 r_{10}}}=\sum_{j=0}^{2} \sum_{i=1}^{m_{j}} t_{i j k} \lambda_{j k F C} \lambda_{j k} z_{9 i j k r_{9}} z_{10 i j k r_{10}}$

(xv) $\frac{\partial^{2} L}{\partial \beta_{10 r_{10}} \partial \beta_{10 r_{10}^{\prime}}}=\sum_{j=0}^{2} \sum_{i=1}^{m_{j}}\left[d_{i j k}+\lambda_{j k}\left(v_{i j k}-h_{i j k}-q_{i j k}-t_{i j k}\right)\right] \lambda_{j k} z_{10 i j k r_{10}} z_{10 i j k r_{10}^{\prime}}$

com $v_{i j k}=P_{2222}-P_{2111}-P_{1211}-P_{1121}-P_{1112}$

$$
+P_{1111}\left(P_{1000}+P_{0100}+P_{0010}+P_{0001}-P_{1111}\right)
$$

$r_{s}, r_{s}^{\prime}=1, \ldots, R_{s} ; \mathrm{s}=6, \ldots, 10 ;$ que em notação matricial podem ser expressas como:

$$
\begin{gathered}
\frac{\partial^{2} L}{\partial \boldsymbol{\beta}_{6} \boldsymbol{\beta}_{6}^{\prime}}=\boldsymbol{Z}_{6}^{\prime}\left[\mathbf{A}^{*}+\boldsymbol{L}_{I} \mathbf{E}\right] \boldsymbol{L}_{I} \boldsymbol{Z}_{6}, \frac{\partial^{2} L}{\partial \boldsymbol{\beta}_{6} \boldsymbol{\beta}_{7}^{\prime}}=\boldsymbol{Z}_{6}^{\prime} \mathbf{F} \boldsymbol{L}_{I} \boldsymbol{L}_{F S} \boldsymbol{Z}_{7} \\
\frac{\partial^{2} L}{\partial \boldsymbol{\beta}_{6} \boldsymbol{\beta}_{8}^{\prime}}=\boldsymbol{Z}_{6}^{\prime}\left[\boldsymbol{L}_{F S} \mathbf{F}+\boldsymbol{L}_{F C} \mathbf{G}\right] \boldsymbol{L}_{I} \boldsymbol{Z}_{8}, \frac{\partial^{2} L}{\partial \boldsymbol{\beta}_{6} \boldsymbol{\beta}_{9}^{\prime}}=\boldsymbol{Z}_{6}^{\prime} \mathbf{G} \boldsymbol{L}_{I} \boldsymbol{L}_{F C} \boldsymbol{Z}_{9}, \frac{\partial^{2} L}{\partial \boldsymbol{\beta}_{6} \boldsymbol{\beta}_{10}^{\prime}}=\boldsymbol{Z}_{6}^{\prime} \mathbf{H} \boldsymbol{L}_{I} \mathbf{L} \boldsymbol{Z}_{10} \\
\frac{\partial^{2} L}{\partial \boldsymbol{\beta}_{7} \boldsymbol{\beta}_{7}^{\prime}}=\boldsymbol{Z}_{7}^{\prime}\left[\mathbf{B}^{*}+\boldsymbol{L}_{F S} \mathbf{M}\right] \boldsymbol{L}_{F S} \boldsymbol{Z}_{7}, \frac{\partial^{2} L}{\partial \boldsymbol{\beta}_{7} \boldsymbol{\beta}_{8}^{\prime}}=\boldsymbol{Z}_{7}^{\prime}\left[\mathbf{B}^{*}+\boldsymbol{L}_{F S} \mathbf{M}+\boldsymbol{L}_{F C} \mathbf{N}\right] \boldsymbol{L}_{F S} \boldsymbol{Z}_{8} \\
\frac{\partial^{2} L}{\partial \boldsymbol{\beta}_{7} \boldsymbol{\beta}_{9}^{\prime}}=\boldsymbol{Z}_{7}^{\prime} \mathbf{N} \boldsymbol{L}_{F S} \boldsymbol{L}_{F C} \boldsymbol{Z}_{9}, \frac{\partial^{2} L}{\partial \boldsymbol{\beta}_{7} \boldsymbol{\beta}_{10}^{\prime}}=\boldsymbol{Z}_{7}^{\prime} \mathbf{Q} \boldsymbol{L}_{F S} \mathbf{L} \boldsymbol{Z}_{10} \\
\frac{\partial^{2} L}{\partial \boldsymbol{\beta}_{8} \boldsymbol{\beta}_{8}^{\prime}}=\boldsymbol{Z}_{8}^{\prime}\left[\boldsymbol{L}_{F S}\left(\mathbf{M} \boldsymbol{L}_{F S}+\mathbf{B}^{*}+\mathbf{S} \boldsymbol{L}_{F C}\right)+\boldsymbol{L}_{F C}\left(\mathbf{N} \boldsymbol{L}_{F S}+\mathbf{C}^{*}+\mathbf{R} \boldsymbol{L}_{F C}\right)\right] \boldsymbol{Z}_{8}
\end{gathered}
$$




$$
\begin{gathered}
\frac{\partial^{2} L}{\partial \boldsymbol{\beta}_{8} \boldsymbol{\beta}_{9}^{\prime}}=\boldsymbol{Z}_{8}^{\prime}\left[\mathbf{N} \boldsymbol{L}_{F S}+\mathbf{C}^{*}+\mathbf{R} \boldsymbol{L}_{F C}\right] \boldsymbol{L}_{F C} \boldsymbol{Z}_{9}, \frac{\partial^{2} L}{\partial \boldsymbol{\beta}_{8} \boldsymbol{\beta}_{10}^{\prime}}=\boldsymbol{Z}_{8}^{\prime}\left[\mathbf{Q} \boldsymbol{L}_{F S}+\mathbf{T} \boldsymbol{L}_{F C}\right] \mathbf{L} \boldsymbol{Z}_{10}, \\
\frac{\partial^{2} L}{\partial \boldsymbol{\beta}_{9} \boldsymbol{\beta}_{9}^{\prime}}=\boldsymbol{Z}_{9}^{\prime}\left[\mathbf{C}^{*}+\mathbf{U} \boldsymbol{L}_{F C}\right] \boldsymbol{L}_{F C} \boldsymbol{Z}_{9}, \frac{\partial^{2} L}{\partial \boldsymbol{\beta}_{9} \boldsymbol{\beta}_{10}^{\prime}}=\boldsymbol{Z}_{9}^{\prime} \mathbf{T} \boldsymbol{L}_{F C} \mathbf{L} \boldsymbol{Z}_{10}, \\
\text { e } \frac{\partial^{2} L}{\partial \boldsymbol{\beta}_{10} \boldsymbol{\beta}_{10}^{\prime}}=\boldsymbol{Z}_{10}^{\prime}\left[\mathbf{D}^{*}+\mathbf{L}(\mathbf{V}-\mathbf{H}-\mathbf{Q}-\mathbf{T})\right] \mathbf{L} \boldsymbol{Z}_{10}
\end{gathered}
$$

com:

$$
\begin{aligned}
& \mathbf{A}^{*}=\operatorname{diag}\left(a_{i j k}\right), \mathbf{B}^{*}=\operatorname{diag}\left(b_{i j k}\right), \mathbf{C}^{*}=\operatorname{diag}\left(c_{i j k}\right), \mathbf{D}^{*}=\operatorname{diag}\left(d_{i j k}\right), \\
& \mathbf{E}=\operatorname{diag}\left(e_{i j k}\right), \mathbf{F}=\operatorname{diag}\left(f_{i j k}\right), \mathbf{G}=\operatorname{diag}\left(g_{i j k}\right), \mathbf{H}=\operatorname{diag}\left(h_{i j k}\right), \\
& \mathbf{M}=\operatorname{diag}\left(m_{i j k}\right), \mathbf{N}=\operatorname{diag}\left(n_{i j k}\right), \mathbf{Q}=\operatorname{diag}\left(q_{i j k}\right), \mathbf{R}=\operatorname{diag}\left(r_{i j k}\right), \\
& \mathbf{S}=\operatorname{diag}\left(s_{i j k}\right), \mathbf{T}=\operatorname{diag}\left(t_{i j k}\right), \mathbf{U}=\operatorname{diag}\left(u_{i j k}\right), \mathbf{V}=\operatorname{diag}\left(v_{i j k}\right) ;
\end{aligned}
$$

$P_{u v x y}, \mathbf{Z}_{s}, s=6, \ldots, 10, \boldsymbol{L}_{I}, \boldsymbol{L}_{F S}, \boldsymbol{L}_{F C}$ e $\mathbf{L}$ definidos como anteriormente. 


\section{Apêndice C}

\section{Códigos dos processos iterativos}

\section{C.1 Modelo Beta-Binomial}

O código para o modelo implementado no pacote S-Plus encontra-se na página http://www.ime.usp.br/ mayra e pode ser utilizado para qualquer estudo para o qual se deseja ajustar um modelo Beta-Binomial para probabilidade de acerto. Para tanto, devese disponibilizar um banco de dados com as contagens de acertos (chamada de "acerto") e totais (chamada de "total"), e com as variáveis explicativas a serem discriminadas nas matrizes "Z1"e "Z2". É necessário também explicitar com os valores inicias dos parâmetros nos vetores "beta1"e "beta2".

A saída do programa é composta pelo número de iterações para atingir a convergência (h), o valor do logaritmo da verossimilhança (L), os valores estimados dos parâmetros (beta1, beta2) e a matriz de covariâncias desses parâmetros (variancia).

\section{C.2 Modelos Poisson Tetravariado completo (3.1) e final (3.2)}

Os códigos para os dois modelos implementados no pacote S-Plus encontram-se na página http://www.ime.usp.br/ mayra e podem ser utilizados para qualquer estudo para o qual se deseje ajustar um modelo Poisson Tetravariado com as características dos modelos (3.1) e (3.2). 
Para utilizar o código do modelo (3.1) deve-se disponibilizar um banco de dados com as contagens de totais na avaliação inicial (seqüências treinada e controle) e final (sequiências treinada e controle), variáveis chamadas respectivamente de "inicseq", "iniccont", "finseq"e "fincont"; além das variáveis explicativas a serem discriminadas nas matrizes "Z1", "Z2", "Z3", "Z4"e "Z5". É necessário também explicitar com os valores inicias dos parâmetros nos vetores "beta1", "beta2", "beta3", "beta4"e "beta5".

A saída do programa é composta pelo número de iterações para atingir a convergência (k), o valor do logaritmo da verossimilhança ( $\mathrm{LV}$ ), os valores estimados dos parâmetros (beta1, beta2, beta3, beta4 e beta5) e a matriz de covariâncias desses parâmetros (variancia).

Para o modelo (3.2), o banco de dados deve ser o mesmo. Deve-se especificar as variáveis explicativas nas matrizes "Z6", "Z7", "Z8", "Z9"e "Z10" de acordo com (3.2), assim como os valores iniciais dos parâmetros nos vetores "beta6", "beta7", "beta8", "beta9"e "beta10". As saídas são análogas às do código do modelo (3.1) 
Apêndice D

Resultados das simulações 
Tabela D.1: Resultados das simulações para o modelo Beta-Binomial com amostras de tamanhos 50, 100 e 200

\begin{tabular}{|c|c|c|c|c|c|c|c|c|}
\hline & & & \multicolumn{2}{|c|}{$\mathrm{n}=50$} & \multicolumn{2}{|c|}{$\mathrm{n}=100$} & \multicolumn{2}{|c|}{$\mathrm{n}=200$} \\
\hline $\begin{array}{l}\text { Parâ- } \\
\text { metro }\end{array}$ & $\begin{array}{c}\text { Valor } \\
\text { original }\end{array}$ & $\begin{array}{l}\text { Simu- } \\
\text { lação }\end{array}$ & $\begin{array}{l}\text { Esti- } \\
\text { mativa }\end{array}$ & $\begin{array}{l}\text { Vari- } \\
\text { ação }\end{array}$ & $\begin{array}{l}\text { Esti- } \\
\text { mativa }\end{array}$ & $\begin{array}{l}\text { Vari- } \\
\text { ação }\end{array}$ & $\begin{array}{l}\text { Esti- } \\
\text { mativa }\end{array}$ & $\begin{array}{l}\text { Vari- } \\
\text { ação }\end{array}$ \\
\hline \multirow[t]{6}{*}{$\beta_{10}$} & 2,20 & 1 & 2,05 & $-7 \%$ & 2,21 & $0,5 \%$ & 2,08 & $-5 \%$ \\
\hline & & 2 & 1,79 & $-18 \%$ & 2,21 & $0,5 \%$ & 2,33 & $6 \%$ \\
\hline & & 3 & 2,41 & $9 \%$ & 2,13 & $-3 \%$ & 2,11 & $-4 \%$ \\
\hline & & 4 & 2,22 & $1 \%$ & 2,03 & $-8 \%$ & 2,23 & $1 \%$ \\
\hline & & 5 & 2,13 & $-3 \%$ & 2,35 & $7 \%$ & 2,12 & $-4 \%$ \\
\hline & & média & 2,12 & $-4 \%$ & 2,19 & $-0,5 \%$ & 2,17 & $-1 \%$ \\
\hline \multirow[t]{6}{*}{$\beta_{11}$} & $-2,60$ & 1 & $-2,74$ & $5 \%$ & $-2,49$ & $-4 \%$ & $-2,39$ & $-8 \%$ \\
\hline & & 2 & $-2,14$ & $-18 \%$ & $-2,97$ & $14 \%$ & $-2,56$ & $-2 \%$ \\
\hline & & 3 & $-2,57$ & $-1 \%$ & $-2,58$ & $-1 \%$ & $-2,48$ & $-5 \%$ \\
\hline & & 4 & $-2,54$ & $-2 \%$ & $-2,53$ & $-3 \%$ & $-2,85$ & $10 \%$ \\
\hline & & 5 & $-2,22$ & $-15 \%$ & $-2,64$ & $1 \%$ & $-2,94$ & $13 \%$ \\
\hline & & média & $-2,44$ & $-6 \%$ & $-2,64$ & $1 \%$ & $-2,64$ & $1 \%$ \\
\hline \multirow[t]{6}{*}{$\beta_{20}$} & $-1,20$ & 1 & $-1,46$ & $22 \%$ & $-1,07$ & $-11 \%$ & $-1,26$ & $5 \%$ \\
\hline & & 2 & $-0,72$ & $-40 \%$ & $-1,44$ & $20 \%$ & $-1,21$ & $1 \%$ \\
\hline & & 3 & $-0,82$ & $-32 \%$ & $-1,00$ & $-17 \%$ & $-1,29$ & $8 \%$ \\
\hline & & 4 & $-0,81$ & $-33 \%$ & $-1,06$ & $-12 \%$ & $-1,14$ & $-5 \%$ \\
\hline & & 5 & $-1,58$ & $32 \%$ & $-1,50$ & $25 \%$ & $-1,22$ & $2 \%$ \\
\hline & & média & $-1,08$ & $-10 \%$ & $-1,21$ & $1 \%$ & $-1,22$ & $2 \%$ \\
\hline \multirow[t]{6}{*}{$\beta_{21}$} & 1,90 & 1 & 1,37 & $-28 \%$ & 1,92 & $1 \%$ & 2,00 & $5 \%$ \\
\hline & & 2 & 1,56 & $-18 \%$ & 2,31 & $22 \%$ & 2,10 & $11 \%$ \\
\hline & & 3 & 1,51 & $-21 \%$ & 1,56 & $-18 \%$ & 2,00 & $5 \%$ \\
\hline & & 4 & 1,45 & $-24 \%$ & 1,65 & $-13 \%$ & 1,77 & $-7 \%$ \\
\hline & & 5 & 2,37 & $25 \%$ & 2,55 & $34 \%$ & 1,92 & $1 \%$ \\
\hline & & média & 1,65 & $-13 \%$ & 2,00 & $5 \%$ & 1,96 & $3 \%$ \\
\hline
\end{tabular}


Tabela D.2: Resultados das simulações para o modelo Poisson Tetravariado com amostras de tamanhos 50, 100 e 200

\begin{tabular}{|c|c|c|c|c|c|c|c|c|}
\hline & & & \multicolumn{2}{|c|}{$\mathrm{n}=50$} & \multicolumn{2}{|c|}{$\mathrm{n}=100$} & \multicolumn{2}{|c|}{$\mathrm{n}=200$} \\
\hline $\begin{array}{l}\text { Parâ- } \\
\text { metro }\end{array}$ & $\begin{array}{c}\text { Valor } \\
\text { original }\end{array}$ & $\begin{array}{l}\text { Simu- } \\
\text { lação }\end{array}$ & $\begin{array}{l}\text { Esti- } \\
\text { mativa }\end{array}$ & $\begin{array}{l}\text { Vari- } \\
\text { ação }\end{array}$ & $\begin{array}{l}\text { Esti- } \\
\text { mativa }\end{array}$ & $\begin{array}{l}\text { Vari- } \\
\text { ação }\end{array}$ & $\begin{array}{l}\text { Esti- } \\
\text { mativa }\end{array}$ & $\begin{array}{l}\text { Vari- } \\
\text { ação }\end{array}$ \\
\hline \multirow[t]{6}{*}{$\beta_{10}$} & 3,22 & 1 & 3,27 & $2 \%$ & 3,23 & $0,3 \%$ & 3,21 & $-0,3 \%$ \\
\hline & & 2 & 3,22 & $0 \%$ & 3,27 & $2 \%$ & 3,22 & $0 \%$ \\
\hline & & 3 & 3,21 & $-0,3 \%$ & 3,21 & $-0,3 \%$ & 3,19 & $-1 \%$ \\
\hline & & 4 & 3,21 & $-0,3 \%$ & 3,23 & $0,3 \%$ & 3,19 & $-1 \%$ \\
\hline & & 5 & 3,18 & $-1 \%$ & 3,19 & $-1 \%$ & 3,20 & $-1 \%$ \\
\hline & & média & 3,22 & $0 \%$ & 3,23 & $0,3 \%$ & 3,21 & $-0,3 \%$ \\
\hline \multirow[t]{6}{*}{$\beta_{11}$} & 0,11 & 1 & 0,05 & $-55 \%$ & 0,07 & $-36 \%$ & 0,12 & $9 \%$ \\
\hline & & 2 & 0,16 & $45 \%$ & 0,14 & $27 \%$ & 0,16 & $45 \%$ \\
\hline & & 3 & 0,14 & $27 \%$ & 0,11 & $0 \%$ & 0,14 & $27 \%$ \\
\hline & & 4 & 0,17 & $55 \%$ & 0,09 & $-18 \%$ & 0,12 & $9 \%$ \\
\hline & & 5 & 0,20 & $82 \%$ & 0,13 & $18 \%$ & 0,13 & $18 \%$ \\
\hline & & média & 0,14 & $27 \%$ & 0,11 & $0 \%$ & 0,13 & $18 \%$ \\
\hline \multirow[t]{6}{*}{$\beta_{20}$} & 2,89 & 1 & 2,87 & $-1 \%$ & 2,89 & $0 \%$ & 2,91 & $1 \%$ \\
\hline & & 2 & 2,92 & $1 \%$ & 2,91 & $1 \%$ & 2,87 & $-1 \%$ \\
\hline & & 3 & 2,90 & $0,3 \%$ & 2,88 & $-0,3 \%$ & 2,88 & $-0,3 \%$ \\
\hline & & 4 & 2,88 & $-0,3 \%$ & 2,94 & $2 \%$ & 2,83 & $-2 \%$ \\
\hline & & 5 & 2,96 & $2 \%$ & 2,82 & $-2 \%$ & 2,88 & $-0,3 \%$ \\
\hline & & média & 2,91 & $1 \%$ & 2,89 & $0 \%$ & 2,87 & $-1 \%$ \\
\hline \multirow[t]{6}{*}{$\beta_{21}$} & 0,11 & 1 & 0,16 & $45 \%$ & 0,07 & $-36 \%$ & 0,12 & $9 \%$ \\
\hline & & 2 & 0,12 & $9 \%$ & 0,08 & $-27 \%$ & 0,12 & $9 \%$ \\
\hline & & 3 & 0,16 & $45 \%$ & 0,12 & $9 \%$ & 0,13 & $18 \%$ \\
\hline & & 4 & 0,13 & $18 \%$ & 0,03 & $-73 \%$ & 0,19 & $73 \%$ \\
\hline & & 5 & 0,04 & $-64 \%$ & 0,14 & $27 \%$ & 0,07 & $-36 \%$ \\
\hline & & média & 0,12 & $9 \%$ & 0,09 & $-18 \%$ & 0,13 & $18 \%$ \\
\hline \multirow[t]{6}{*}{$\beta_{30}$} & 2,71 & 1 & 2,74 & $1 \%$ & 2,68 & $-1 \%$ & 2,69 & $-0,4 \%$ \\
\hline & & 2 & 2,72 & $0,4 \%$ & 2,67 & $-1 \%$ & 2,71 & $0 \%$ \\
\hline & & 3 & 2,78 & $3 \%$ & 2,66 & $-2 \%$ & 2,73 & $1 \%$ \\
\hline & & 4 & 2,71 & $0 \%$ & 2,74 & $1 \%$ & 2,71 & $0 \%$ \\
\hline & & 5 & 2,80 & $3 \%$ & 2,70 & $-0,1 \%$ & 2,71 & $0 \%$ \\
\hline & & média & 2,75 & $1 \%$ & 2,69 & $-0,4 \%$ & 2,71 & $0 \%$ \\
\hline
\end{tabular}




\begin{tabular}{|c|c|c|c|c|c|c|c|c|}
\hline \multirow[b]{2}{*}{$\begin{array}{l}\text { Parâ- } \\
\text { metro }\end{array}$} & \multirow[b]{2}{*}{$\begin{array}{c}\text { Valor } \\
\text { original }\end{array}$} & \multirow[b]{2}{*}{$\begin{array}{l}\text { Simu- } \\
\text { lação }\end{array}$} & \multicolumn{2}{|c|}{$\mathrm{n}=50$} & \multicolumn{2}{|c|}{$\mathrm{n}=100$} & \multicolumn{2}{|c|}{$\mathrm{n}=200$} \\
\hline & & & $\begin{array}{c}\text { Esti- } \\
\text { mativa }\end{array}$ & $\begin{array}{l}\text { Vari- } \\
\text { ação }\end{array}$ & $\begin{array}{c}\text { Esti- } \\
\text { mativa }\end{array}$ & $\begin{array}{l}\text { Vari- } \\
\text { ação }\end{array}$ & $\begin{array}{c}\text { Esti- } \\
\text { mativa }\end{array}$ & $\begin{array}{l}\text { Vari- } \\
\text { ação }\end{array}$ \\
\hline \multirow[t]{6}{*}{$\beta_{31}$} & 0,24 & 1 & 0,24 & $0 \%$ & 0,27 & $13 \%$ & 0,29 & $21 \%$ \\
\hline & & 2 & 0,26 & $8 \%$ & 0,27 & $13 \%$ & 0,25 & $4 \%$ \\
\hline & & 3 & 0,23 & $-4 \%$ & 0,29 & $21 \%$ & 0,23 & $-4 \%$ \\
\hline & & 4 & 0,19 & $-21 \%$ & 0,21 & $-13 \%$ & 0,24 & $0 \%$ \\
\hline & & 5 & 0,09 & $-63 \%$ & 0,19 & $-21 \%$ & 0,22 & $-8 \%$ \\
\hline & & média & 0,20 & $-17 \%$ & 0,25 & $4 \%$ & 0,25 & $4 \%$ \\
\hline \multirow[t]{6}{*}{$\beta_{40}$} & 3,00 & 1 & 2,98 & $-1 \%$ & 3,02 & $1 \%$ & 2,99 & $-0,3 \%$ \\
\hline & & 2 & 2,93 & $-2 \%$ & 3,03 & $1 \%$ & 2,99 & $-0,3 \%$ \\
\hline & & 3 & 3,04 & $1 \%$ & 3,02 & $1 \%$ & 3,02 & $1 \%$ \\
\hline & & 4 & 2,97 & $-1 \%$ & 3,06 & $2 \%$ & 2,98 & $-1 \%$ \\
\hline & & 5 & 3,03 & $1 \%$ & 2,92 & $-3 \%$ & 2,99 & $-0,3 \%$ \\
\hline & & média & 2,99 & $-0,3 \%$ & 3,00 & $0 \%$ & 2,99 & $-0,3 \%$ \\
\hline \multirow[t]{6}{*}{$\beta_{41}$} & 0,22 & 1 & 0,29 & $32 \%$ & 0,20 & $-9 \%$ & 0,24 & $9 \%$ \\
\hline & & 2 & 0,30 & $36 \%$ & 0,18 & $-18 \%$ & 0,23 & $5 \%$ \\
\hline & & 3 & 0,16 & $-27 \%$ & 0,18 & $-18 \%$ & 0,21 & $-5 \%$ \\
\hline & & 4 & 0,29 & $32 \%$ & 0,15 & $-32 \%$ & 0,22 & $0 \%$ \\
\hline & & 5 & 0,19 & $-14 \%$ & 0,34 & $55 \%$ & 0,21 & $-5 \%$ \\
\hline & & média & 0,25 & $14 \%$ & 0,21 & $-5 \%$ & 0,22 & $0 \%$ \\
\hline \multirow[t]{6}{*}{$\beta_{50}$} & 1,79 & 1 & 1,92 & $7 \%$ & 1,77 & $-1 \%$ & 0,43 & $-76 \%$ \\
\hline & & 2 & 1,78 & $-1 \%$ & 0,75 & $-58 \%$ & 1,58 & $-12 \%$ \\
\hline & & 3 & 2,01 & $12 \%$ & 0,81 & $-55 \%$ & 1,06 & $-41 \%$ \\
\hline & & 4 & 0,83 & $-54 \%$ & 0,72 & $-60 \%$ & 0,98 & $-45 \%$ \\
\hline & & 5 & 1,72 & $-4 \%$ & 1,03 & $-42 \%$ & 0,98 & $-45 \%$ \\
\hline & & média & 1,65 & $-8 \%$ & 1,02 & $-43 \%$ & 1,01 & $-44 \%$ \\
\hline \multirow[t]{6}{*}{$\beta_{51}$} & 0,69 & 1 & 0,01 & $-98 \%$ & 0,31 & $-55 \%$ & 1,28 & $86 \%$ \\
\hline & & 2 & 0,27 & $-61 \%$ & 1,06 & $54 \%$ & 0,33 & $-52 \%$ \\
\hline & & 3 & 0,58 & $-16 \%$ & 0,95 & $38 \%$ & 0,90 & $30 \%$ \\
\hline & & 4 & 1,10 & $59 \%$ & 0,98 & $42 \%$ & 0,80 & $16 \%$ \\
\hline & & 5 & 0,08 & $-88 \%$ & 0,41 & $-41 \%$ & 0,73 & $6 \%$ \\
\hline & & média & 0,41 & $-41 \%$ & 0,74 & $7 \%$ & 0,81 & $17 \%$ \\
\hline
\end{tabular}




\section{Referências Bibliográficas}

[1] Aitchison, J., Ho, C. (1989). The multivariate Poisson log-normal distribution. Biometrika 76, 643-653.

[2] Brooks, S. P., Morgan, J. T., Ridout, M. S., Pack, S. E. (1997). Finite Mixture Models for Proportions. Biometrics 53, 1097-1115.

[3] Diggle, P. J., Liang, K. Y., Zeger, S. (2001). Analysis of Longitudinal Data. New York: Oxford Science Publications.

[4] Everitt, B. S. (1994). A Handbook of Statistical Analysis using S-Plus. London: Chapman and Hall.

[5] Gange, S. J., Munoz, A., Saez, M., Alonso, J. (1996). Use of the Beta-Binomial Distribution to Model the Effect of Policy Changes on Appropriateness of Hospital Stays. Applied Statistics 45, 371-382.

[6] Griffiths, D. A. (1973). Maximum Likelihood Estimation for the Beta-Binomial Distribution and an Application to the Total Number of Cases of a Disease. Biometrics 29, 673-648.

[7] Ho, L. L., Singer, J. M. (1997). Regression Models for Bivariate Counts. Brazilian Journal of Probability and Statistics 11, 175-197.

[8] Ho, L. L., Singer, J. M. (2001). Generalized Least Squares Methods for Bivariate Poisson Regression. Communications in Statistics 30, 263-278.

[9] Holgate, P. (1964). Estimation for the Bivariate Poisson Distribution. Biometrika 51, 241-245. 
[10] Johnson, N. L., Kotz, S. (1969). Distributions in Statistics: discrete distributions. Boston: Houghton Mifflin.

[11] Johnson, N. L., Kotz, S. (1970). Distributions in Statistics: continuous univariate distributions 2. Boston: Houghton Mifflin.

[12] Kawamura, K. (1979). The Structure of Multivariate Poisson Distribution. Kodai Mathematical Journal 2, 337-345.

[13] Kocherlakota, S., Kocherlakota, K. (2001). Regression in the Bivariate Poisson Distribution. Communications in Statistics 30, 815-825.

[14] Singer, J. M., Magalhães, M. N., Petri, C. e Aguemi, R. (2002). Relatório de Análise Estatística sobre o Projeto: Característica do Aprendizado Motor na Doença de Parkinson. Centro de Estatística Aplicada, Departamento de Estatística do Instituto de Matemática e Estatística da Universidade de São Paulo, São Paulo.

[15] Thisted, R. A. (1988). Elements of Statistical Computing: numerical computation. New York: Chapman and Hall.

[16] Williams, D. A. (1975). The Analysis of Binary responses from Toxicological Experiments Involving Reproduction and Teratogenicity. Biometrics 31, 949-952.

[17] Zhu, J., Eickhoff, J. C., Kaiser, M. S. (2003). Modeling the Dependence between Number of Trials and Sucess Probability in Beta-Binomial-Poisson Mixture Distributions. Biometrics 59, 955-961. 